\title{
Scarcity, regulation and endogenous
}

\section{technical progress ${ }^{1}$}

\author{
Raouf Boucekkine $^{2}$, Natali Hritonenko ${ }^{3}$ and Yuri Yatsenko ${ }^{4}$
}

\begin{abstract}
We consider a firm with the following characteristics: (i) it has a vintage capital technology with two complementary factors, capital and a resource input subject to quota; (ii) the resource is increasingly scarce through an exogenously rising price, (iii) scrapping of obsolete capital is endogenous; (iv) technological progress allows saving the regulated input and is endogenous through R\&D investment; (v) the innovation rate increases with R\&D investment and decreases with complexity; (vi) the firm is price-taker and liquidity-constrained. We show that there exists a threshold level for the growth rate of the resource price above which the firm will collapse. Below this threshold, two important properties are found out. In the long-run, a sustainable growth is possible at a growth rate which is independent of the resource price. In the short-run, not only will the firms respond to increasing resource price by increasing R\&D on average, but they will also reduce capital expenditures and speed up the scrapping of older capital goods. Finally, we identify optimal intensive Vs extensive transitional growth regimes depending on the history of the firms.
\end{abstract}

Keywords: Sustainability, Scarcity, environmental regulation, vintage capital, technological progress, dynamic optimization

JEL numbers: C61, D21, D92, O33, Q01

\footnotetext{
${ }^{1}$ We would like to thank Thierry Bréchet, Lucas Bretschger, Paolo Brito, David Cuberes, David de la Croix, Frédéric Docquier, Johan Eyckmans, Gustav Feichtinger, Hans Gersbach, Peter Kort, Omar Licandro, Aude Pommeret, Francesco Ricci, Thomas Rutherford, Robert Tamura, Henry Tulkens, and participants at the 2007 Third Vienna Vintage Workshop, the 2008 MIMI Workshop in Lille (France), and seminars held at ECTH, Zurich, and CORE, Louvain-la-Neuve, for invaluable comments on previous versions. The paper was partially written when Yatsenko visited CORE. Boucekkine acknowledges the financial support of the Belgian research programmes PAI P5/10 and ARC 'Sustainability'. The usual disclaimer applies.

${ }^{2}$ Corresponding author: CORE, Université catholique de Louvain, Belgium, and University of Glasgow, UK. Place Montesquieu, 3, 1348 Louvain-la-Neuve, Belgium. Raouf.Boucekkine@uclouvain.be

${ }^{3}$ Prairie View A\&M University, USA. NaHritonenko@pvamu.edu

${ }^{4}$ Houston Baptist University, USA. YYatsenko@hbu.edu
} 


\section{Introduction}

A crucial issue repeatedly addressed in the ongoing debate on sustainable development is the possibility for the economies to keep on growing while confronted to physical limits and legal constraints such like those related to the limited availability or regenerative capacity of natural resources (fossil energy, fish, forest, etc.), to economic and ecological regulation (emission quotas, harvesting quotas, etc.), or to financial resource constraints at the firm or national economy level. One of the common ideas turns out to be that such a growth possibility is certainly widely open if the economies are able to maintain a permanent stream of innovations, assuring long-term technological progress (see Arrow et al., 2004, for a comprehensive view of sustainability).

In terms of economic theory, the issue actually traces back to seminal studies on the relationship between resource scarcity and innovation. Scarcer resources are increasingly expensive, and this should in a way affect the behavior of consumers and firms and end up shaping the direction of technological progress. A related fundamental hypothesis, popularized by Hicks (1932), is the so-called induced-innovation hypothesis. According to this hypothesis, the change of relative prices of production inputs stimulates innovation directed to save the production factor that becomes relatively expensive. In the context of the energy consumption debate, this hypothesis simply stipulates that in periods of rapidly rising energy prices (relative to other inputs), economic agents will find it more profitable to develop alternative technologies, that is, energy-saving technologies. In their well-known work on the menu of home appliances available for sale in the US (between 1958 and 1993), Newell, Jaffee, and Stavins (1999) concluded that a large portion of energy efficiency improvements in US manufacturing seems to be autonomous, and therefore not driven by the Hicksian mechanism outlined above. However, they also concluded that a non-negligible part of the observed improvement can be attributed to price changes and to the emergence of new energy-efficiency standards, ultimately leading to the elimination of old models.

Indeed, just like scarcity, regulation can also be a decisive determinant of technological progress. As an immediate illustration of such a potential nexus, environmental economists use to put forward the so-called Porter hypothesis (Porter, 1991) according to 
which a carefully designed environmental regulation can increase firm competitiveness by encouraging innovation in environmental technologies. A considerable amount of studies has been devoted to the empirical corroboration of this hypothesis, reaching distinct and contrasted conclusions (see Parto and Herbert-Copley, 2007, for an excellent compilation of case studies).

In this paper, we take the firm perspective, which is the traditional framework for the study of Hicksian technical progress (see among others, the seminal paper of Kamien and Schwartz, 1969). Firms are typically affected by several institutional and economic factors, notably by competition, credit constraints, and legal constraints which are not only linked to ecological regulation. We consider the worst scenario possible in this respect: (1) no market power (the firm is price-taker), (2) liquidity-constraints (the firm cannot incur in a negative cash flow at any date), (3) a quota constraint on the use of a resource input (fossil energy or natural resource like fish as immediate examples), which may feature emission or extraction quotas, (4) the price of this production input is increasing reflecting scarcity, and (5) no substitution is possible between this resource and other production inputs. In such a context, could the firm experience a sustainable growth of profits?

Answering this question properly requires accounting for a comprehensive set of modernization instruments that the firm can use in response to the above constraints. At the first place, the role of innovation and technology adoption at the firm and/or industry level is a key. If the firms do effectively respond to the latter constraints and circumstances by doing more $\mathrm{R} \& \mathrm{D}$ and/or adopting better technologies, then the "sustainability problem", stated in the beginning, can be at least partially solved. But firms cannot always push on this command button for many reasons. Two are quite obvious. First of all, firms are subject to financial or liquidity constraints, as mentioned above. If the firms do not face any type of financial constraints, then they could finance R\&D expenditures and/or technology adoption with no limit, which is certainly unrealistic. Second, technological complexity can be a decisive factor. It is very well known that the success of $\mathrm{R} \& \mathrm{D}$ and technology transfer programs depends, among others, on the complexity and sophistication of the technologies to be up-graded (see for example, Segerstrom, 2000). Needless to say, the problem of technological sophistication 
is also a sensitive barrier to technological progress because of limited amount of available skills and hi-tech capital. We shall account for it in our modeling.

In addition, to innovative and/or adoptive $R \& D$, firms may decide to scrap old and definitely non-sustainable technologies with their associated capital goods and to replace them (or not) with leading technologies and new equipment. If one aims to thoroughly capture the mechanisms of modernization, the latter instruments are crucial to consider. Typically, firms will respond by combining all these instruments and by choosing the optimal timing for each of them. We take this avenue here by considering vintage technologies at the firm level, allowing the firm to innovate, to scrap, and to invest. A fundamental contribution of this paper is the identification of the optimal modernization strategies pursued by firms based on the three instruments listed just above.

We shall use vintage capital technologies in line with Malcomson (1975), Benhabib and Rustichini (1991), Boucekkine et al. (1997, 1999) and Hritonenko and Yatsenko (1996, 2005). There are two inputs, capital and a resource subject to quota, which can be fossil energy or any natural resource. Capital goods produced at different dates embody different technologies, the youngest vintages are the most resource-saving. Beside realism, working with vintage capital production functions allows us to capture some key elements of the problem under consideration, which would be lost under the typical assumption of homogenous capital. For instance, facing an emission tax, firms are tempted to downsize. However, in the vintage capital framework where the firm also chooses the optimal age structure of capital, downsizing entails modernization: the oldest and, thus, the least efficient technologies are then removed.

\section{Main contributions}

Our paper essentially makes three contributions:

i) Within a realistic (and thus sophisticated) firm framework, it characterizes finely the inducement mechanisms at work. Essentially, our work identifies a highly nonlinear inducement mechanism. In particular, there exists a threshold level for the growth rate of the resource price above which the firm will collapse: for these price values, the inducement mechanism does not even make sense. Below this threshold, two important properties are found out. In the long-run, sustainable growth regimes are possible but within such regimes, 
the growth rate of technological progress is independent of the resource price. In our work, this is a long-term property, which occurs when the resource price does not grow too much. In the short-run, the inducement mechanism seems to work: not only will the firms respond to increasing resource price by increasing resource-saving investment on average, they will also reduce capital expenditures and speed up the scrapping of older (and more resource consuming) capital goods, which is highly consistent with the evidence gathered by Newell et al. (1999).

ii) Secondly, our paper makes a contribution to the literature of growth under scarcity and regulation, which is an important component of the modern environmental economics literature. Among the many recent papers on the topic, Tsur and Zemel's contributions $(2003,2005)$ are of particular interest since they carefully derive the possible dynamics arising under scarcity and endogenous technological progress (via the development of backstop substitutes in these papers). With respect to this literature, our contribution is double. On one hand, since both scarcity and regulation are considered, it is possible to study which one is more harmful (if any) to growth. For constant resource prices (no scarcity), we show that sustainable growth regimes are always possible thanks to endogenous technical progress under non-increasing input quotas and despite liquidity constraints. Under scarcity, this property holds as long as the growth rate of the resource price is below a threshold. Above this threshold, no sustainable growth is possible. A second contribution relies on the optimal transition dynamics derived. We disentangle 3 different optimal dynamic patterns: economic collapse (originating in too high energy or capital prices), intensive growth (sustained investment in new capital and in R\&D with scrapping of the oldest capital goods), and extensive growth (sustained investment in new capital and in R\&D without scrapping of the oldest capital goods). In particular, our paper is the first one to disentangle the last transition regime as a possible optimal regime.

iii) Last but not least, the contribution is technical. To our knowledge, this is the first paper with vintage capital, endogenous scrapping, and endogenous technological progress (see next paragraph for more details). The technical 
difficulties are numerous but we manage to find a way to bring out a fine enough analytical characterization of optimal paths.

\section{Relation to the literature}

Our paper contributes to the literature of vintage capital models. Due to the analytical complexity of vintage models, very few papers rely on such specifications. A noticeable exception is Feichtinger, Hartl, Kort, and Veliov (2005) who introduced a proper specification of embodied technological progress underlying the considered vintage capital structure. They concluded that if learning costs are incorporated into the analysis (i.e., running new machines at their full productivity potential takes time), then the magnitude of modernization effect is reduced, and regulation has a markedly negative effect on industry profits. Our paper extends the latter result in two important directions: it endogenizes the optimal lifetime of technologies and associated equipment through endogenous scrapping decision and it endogenizes the pace of technological progress in the workplace by considering an optimal innovative or adoptive R\&D decision (the technological progress is exogenous at the firm level in Feichtinger et al. (2005). In such a context, the set of possible modernization strategies is much richer. On the other hand, our paper extends the more traditional vintage literature following Solow et al. (1966), like Boucekkine et al. (1997) or Hritonenko and Yatsenko (1996), by endogenizing technical progress, which definitely enriches the model in many directions as it will be explained along the way. Recently, Hart (2004) has built up a multisectoral endogenous growth model with an explicit vintage sector. Beside the macroeconomic approach taken, this paper differs from ours in many essential respects: there are two types of R\&D, one output-augmenting and the other, say, environmental-friendly, while in our model only resource-saving adoptive and/or innovative $R \& D$ is allowed. In addition, the model of Hart (2004) has no explicit scarcity feature, and the treatment of vintages is rather short (only two exogenously given vintages are considered in the end, no endogenous scrapping incorporated).

On the other hand, our paper is directly related to the literature on technological progress under increasing energy prices and regulation, as surveyed by Jaffe et al. (2002). As explained above, it can be connected to the empirical findings in the field. Beside the 
already mentioned paper of Newell et al. (1999), it can be indeed closely related to the recent important work of Popp (2002). Using energy patent citations, the latter establishes that both energy prices and the quality of the existing knowledge have significantly shaped energy-saving innovations, therefore confirming the relevance of the inducement mechanism in this context. Moreover, Popp also shows clearly that the omission of the existing quality of knowledge negatively affects the estimation results. In our modeling, we do account for the latter feature through the complexity effect $\grave{a}$ la Segerstrom outlined above: thanks to this specification, there is an immediate link between current and future research, as recommended by Popp (2002).

Last but not least, our paper can be also directly connected to the theoretical literature on scarcity and growth originating in the limits to growth stream. In particular, it shares with Tsur and Zemel $(2003,2005)$ the objective to characterize the different possible optimal patterns of technological progress and growth when the resources are increasingly scarce. While the modeling strategies are different (including the modeling of scarcity and the specification of endogenous technical progress and production) the main conclusions are similar in that collapse, and sustainable growth regimes are identified. Our vintage approach and the inclusion of environmental regulation allows for an even more complete characterization of sustainable development possibilities and strategies.

The rest of the paper is organized as follows. Section 2 formally describes our firm optimization problem and outlines some of its peculiarities. Section 3 derives the optimality conditions and interprets them. Section 4 is concerned with the long-term optimal behavior of firms and Section 5 identifies the short-term modernization strategies that the firms pursue in response to regulation and prices. Section 6 concludes.

\section{The firm problem}

We shall consider the problem of a firm seeking to maximize the net profit that takes into account the consumption $E(t)$ of a regulated resource, the investment $R(t)$ to innovative and/or adoptive $\mathrm{R} \& \mathrm{D}$, and the investment $\mu(t)$ into new capital: 


$$
I=\int_{0}^{\infty} e^{-r t}[(1-\theta) Q(t)-p(t) E(t)-R(t)-k(t) \mu(t)] d t \longrightarrow \max _{\mu a, R}
$$

where $k(t)$ is the given unit capital price (per capacity unit), $p(t)$ is the given price of the regulated resource, and $e^{-r t}$ is the discounting factor. We assume that $p(t)=\bar{P} e^{\gamma t}, \quad \gamma \geq 0, \bar{P} \geq 0$, reflecting scarcity of the resource. Then, $Q(t)$ is the total product output at $t$,

$$
\begin{gathered}
Q(t)=\int_{a(t)}^{t} \mu(\tau) d \tau \\
c(t)=(1-\theta) Q(t)-p(t) E(t)-R(t)-k(t) \mu(t)
\end{gathered}
$$

is the net profit or cash flow, $\theta$ is a tax rate on production or sales (which could be also interpreted as an emission tax in the environmental context, see Feichtinger et al., 2005) . We postulate a Leontief vintage capital production function as in Malcomson (1975), Boucekkine, Germain, and Licandro (1997, 1999) or Hritonenko and Yatsenko (1996, 2005). In equation (2), $a(t)$ measures the vintage index of the oldest machine still in use at time $t$, or in other words, $t-a(t)$ is the scrapping time at date $t$. The complexity of the optimization problem considered in this paper comes from the fact that $a$ is a control variable, which is quite unusual in economic theory. We shall come back to this point in detail later. For now, let us notice that we do not assume any output-augmenting (embodied or disembodied) technological progress: whatever the vintage $\tau$ is, all machines produce one unit of output. In our framework, the technological progress is exclusively resource-saving, which is the key component of the debate around technological progress and environmental sustainability.

In contrast to the related literature (notably to Feichtinger et al., 2005, 2006), we assume that firms choose the optimal lifetime of their capital goods, and also invest in adoptive and/or innovative $\mathrm{R} \& \mathrm{D}$. Let us call $\beta(\tau)$ the level of the resource-saving technological progress at date $t$. We postulate that this level evolves endogenously according to:

$$
\frac{\beta^{\prime}(\tau)}{\beta(\tau)}=\frac{f(R(\tau))}{\beta^{d}(\tau)}, \quad d>0,
$$

where $f$ is increasing and concave: $d f / d R>0, d^{2} f / d R^{2}<0$. Equation (4) deserves a few comments. It stipulates that the rate of resource-saving technical progress is an increasing (and concave) function of the R\&D effort and a decreasing function of its level. The latter 
specification is designed to reflect the negative impact of technological complexity on R\&D success. The parameter $d$ measures the extent to which complexity impacts the rate of technological progress (see Segerstrom, 2000, for example). It will play an important role hereafter, consistently with the available evidence on the role of technological complexity in the adoption of new technologies.

We also assume that the resource-saving technological progress is fully embodied in new capital goods, which implies, keeping the Leontief structure outlined above, that total resource consumption is given by

$$
E(t)=\int_{a(t)}^{t} \frac{\mu(\tau)}{\beta(\tau)} d \tau .
$$

Now we introduce the quota constraint on the regulated resource:

$$
E(t) \leq E_{\max }(t),
$$

where the regulation function $E_{\max }(t)$ is given. The firms are also subject to a second type of constraint, financial constraint, which we also model in a straightforward way by imposing the non-negativity of cash-flows, $c(t)$, at any date $t$, as we will see later.

Let us now summarize the optimal control problem to tackle. The unknown functions are:

- the investment $\mu(t), \mu(t) \geq 0$, into new capital (measured in the capacity units)

- the R\&D investment $R(t), R(t) \geq 0$, and the technology $\beta(t)$,

- the capital scrapping time $t-a(t), a^{\prime}(t) \geq 0, a(t)<t$,

- the output $Q(t)$, cash-flow $c(t)$, and resource consumption $E(t), t \in[0, \infty)$.

The constraints are given by the quota (6), the positivity and liquidity constraints, and other regularity conditions:

$$
R(t) \geq 0, \quad c(t) \geq 0, \quad \mu(t) \geq 0, \quad a^{\prime}(t) \geq 0, \quad a(t) \leq t,
$$

The constraint $a^{\prime}(t) \geq 0$ is standard in vintage capital models and implies that scrapped machines cannot be reused. We shall also specify the initial conditions as follows:

$$
a(0)=a_{0}<0, \quad \beta\left(a_{0}\right)=\beta_{0}, \quad \mu(\tau) \equiv \mu_{0}(\tau), R(\tau) \equiv R_{0}(\tau), \quad \tau \in\left[a_{0}, 0\right] .
$$

The optimal control problem (1)-(8) has several mathematical peculiarities. We come back to the technical part in the next Section 3 where the necessary optimality conditions 
are developed. Before, let us start stressing that in our modelling, technological improvements affect only the new capital goods. This is crystal clear in equation (5) giving total resource consumption. Of course, this need not to be the case in general. Part of resource-saving innovations is probably disembodied, and a more general formulation of the problem taking into account this aspect would, in particular, replace the ODE (4) for $\beta(t)$ by a PDE for $\beta(\tau, t)$. This extension is out of the scope of this paper. Second, one would find somehow strange to have imposed scarcity (exogenously increasing price) and a quota on the same input. There are two different responses to this objection. The first one is to defend the realism of such a specification. Suppose this resource input is fossil energy. Then such a resource is typically increasingly scarce, but at the same time, pollution quotas, as originated in international protocols, do imply upper bounds on the use of such an input. Second, we will see rather quickly that scarcity and quota do not have at all the same implications within our set-up so that one can directly figure out that there is no redundancy between the two characteristics. Last but not least, one has to mention that the results obtained in this framework will remain qualitatively the same in an optimal growth set-up with a linear utility function. With nonlinear utility functions, the (already extremely complicated) problem becomes even more trickier due to the endogeneity of the interest rate. We, therefore, choose the firm problem setting. ${ }^{5}$

\section{Extremum conditions}

Let us derive optimality conditions. For mathematical convenience, we change the unknown (decision) variable $\mu(t)$ to

$$
m(t)=\mu(t) / \beta(t)
$$

which is also the investment into new capital (but measured in resource consumption units rather than in capacity units). In the variables $R$ and $m$, the optimization problem (1)-(8) becomes

$$
\begin{aligned}
& I=\int_{0}^{\infty} e^{-r t}[(1-\theta) Q(t)-p(t) E(t)-R(t)-k(t) \beta(t) m(t)] d t \longrightarrow \max _{R, m, a} \\
& c(t)=(1-\theta) Q(t)-p(t) E(t)-R(t)-k(t) \beta(t) m(t),
\end{aligned}
$$

\footnotetext{
${ }^{5}$ The computations for the optimal growth model with linear utility are available upon request.
} 


$$
\begin{gathered}
Q(t)=\int_{a(t)}^{t} \beta(\tau) m(\tau) d \tau, \\
E(t)=\int_{a(t)}^{t} m(\tau) d \tau, \quad E(t) \leq E_{\max }(t), \\
R(t) \geq 0, \quad m(t) \geq 0, \quad c(t) \geq 0, \quad a^{\prime}(t) \geq 0, \quad a(t) \leq t, \\
a(0)=a_{0}<0, \quad \beta\left(a_{0}\right)=\beta_{0}, \quad m(\tau) \equiv m_{0}(\tau), R(\tau) \equiv R_{0}(\tau), \quad \tau \in\left[a_{0}, 0\right] .
\end{gathered}
$$

The substitution (9) removes $\beta(t)$ from equation (5) and adds it to the last term in the functional (10). Equation (4) for the unknown $\beta(t)$ remains the same. In the case $d>0$, the solution of (4) has the form:

$$
\beta(\tau)=\left(d \int_{0}^{\tau} f(R(v)) d v+B^{d}\right)^{1 / d}
$$

where the constant $B=\beta(0)=\left(d \int_{a_{0}}^{0} f\left(R_{0}(v)\right) d v+\beta_{0}{ }^{d}\right)^{1 / d}$ is uniquely determined by the initial conditions (15). From now on, we work with the following explicit specification for endogenous technological progress:

$$
f(R)=b R^{n}, \quad 0<n<1, \quad b>0 .
$$

By (4), this implies that the elasticity of the rate of technological progress with respect to $\mathrm{R} \& \mathrm{D}$ expenditures is constant and equal to $n$. The larger is $n$, the bigger is the efficiency of investing in R\&D.

The optimization problem (OP) (10)-(17) includes seven unknown functions $R, \beta, m, a$, $Q, c$, and $E$ connected by four equalities (11), (12), (13), and (16). Following Hritonenko and Yatsenko (1996) and Yatsenko (2004), we will choose $R, m$, and $a^{\prime}$ as the independent decision variables (controls) of the OP and consider the rest of the unknown functions $\beta, m, a, Q, c$, and $E$ as the dependent (state) variables.

The majority of optimization models of mathematical economics are treated using firstorder conditions for interior trajectories only. In contrast, the nature of the OP (10)-(17) requires taking into account the inequalities $E(t) \leq E_{\max }(t), R(t) \geq 0, m(t) \geq 0, a^{\prime}(t) \geq 0, a(t) \leq t$, and $c(t) \geq 0$ on unknown variables in the constraints (13) and (14). These inequalities have an essential impact on extremum conditions and optimal dynamics and are treated 
differently in the below analysis. The inequalities $R \geq 0$ and $m \geq 0$ are the standard constraints on control variables, which are common in the optimization theory. The nonstandard constraints $a^{\prime}(t) \geq 0$ and $a(t) \leq t$ are handled following the technique developed by Hritonenko and Yatsenko in several papers already cited. The constraint $E \leq E_{\max }$ is considered in two cases of Theorem 1 below. Finally, the constraint $c \geq 0$ is the most inconvenient and is analyzed separately in Section 5 (see also Remark 2 below).

Let the given functions $p, k$, and $E_{\max }$ be continuously differentiable, and $m_{0}$ and $R_{0}$ be continuous. To keep the OP statement correct, the smoothness of the unknown variables should be consistent. We will assume that $R$ and $m$ (and $a^{\prime}$ when necessary) are measurable almost everywhere (a.e.) on $[0, \infty)$. Then, the unknown state variables $a, c, Q$, and $E$ in (10)-(15) are a.e. continuous on $[0, \infty)$, as established in Hritonenko and Yatsenko (2006). We also assume a priori that the improper integral in (10) converges. The necessary condition for an extremum (NCE) in the OP (10)-(17) is given by the following statement

Theorem 1. Let $R^{*}(t), m^{*}(t), a^{*}(t), \beta^{*}(t), Q^{*}(t), c^{*}(t), E^{*}(t), t \in[0, \infty)$, be a solution of the $O P(10)-(17)$.

(A) If $E^{*}(t)=E_{\max }(t)$ and $c^{*}(t)>0$ at $t \in \Delta \subset[0, \infty)$, and $E_{\max }{ }^{\prime}(t) \leq 0$, then

$$
\begin{array}{ll}
I_{R}{ }^{\prime}(t) \leq 0 \text { at } & R^{*}(t)=0, \quad I_{R}{ }^{\prime}(t)=0 \text { at } R^{*}(t)>0, \\
I_{m}{ }^{\prime}(t) \leq 0 \text { at } & m^{*}(t)=0, \quad I_{m}{ }^{\prime}(t)=0 \text { at } m^{*}(t)>0, \quad t \in \Delta,
\end{array}
$$

where

$$
\begin{gathered}
I_{R}{ }^{\prime}(t)=b n R^{n-1}(t) \int_{t}^{\infty} \beta^{1-d}(\tau) m(\tau)\left[\frac{e^{-r \tau}-e^{-r a^{-1}(\tau)}}{r}(1-\theta)-e^{-r \tau} k(\tau)\right] d \tau-e^{-r t}, \\
I_{m}{ }^{\prime}(t)=\int_{t}^{a^{-1}(t)} e^{-r \tau}(1-\theta)[\beta(t)-\beta(a(\tau))] d \tau-e^{-r t} \beta(t) k(t),
\end{gathered}
$$

the state variable $a(t)$ is determined from (13), $a^{-1}(t)$ is the inverse function of $a(t)$, and

$$
\beta(\tau)=\left(d b \int_{0}^{\tau} R^{n}(\xi) d \xi+B^{d}\right)^{\frac{1}{d}} .
$$


(B) If $E^{*}(t)<E_{\max }(t)$ and $c^{*}(t)>0$ at $t \in \Delta$, then

$$
\begin{aligned}
& I_{R}{ }^{\prime}(t) \leq 0 \text { at } R^{*}(t)=0, \quad I_{R^{\prime}}(t)=0 \quad \text { at } R^{*}(t)>0, \\
& I_{m}{ }^{\prime}(t) \leq 0 \text { at } m^{*}(t)=0, \quad I_{m}{ }^{\prime}(t)=0 \text { at } m^{*}(t)>0, \\
& I_{a^{\prime}}(t) \leq 0 \text { at } d a^{*}(t) / d t=0, \quad I_{a^{\prime}}(t)=0 \text { at } d a^{*}(t) / d t>0, \quad t \in \Delta,
\end{aligned}
$$

where

$$
\begin{gathered}
I_{m}{ }^{\prime}(t)=\int_{t}^{a^{-1}(t)} e^{-r \tau}[\beta(t)(1-\theta)-p(\tau)] d \tau-e^{-r t} \beta(t) k(t), \\
I_{a^{\prime}}{ }^{\prime}(t)=\int_{t}^{\infty} e^{-r \tau}[p(\tau)-(1-\theta) \beta(a(\tau))] m(a(\tau)) d \tau,
\end{gathered}
$$

$I_{R}{ }^{\prime}(t)$ is as in (20), and $\beta(t)$ is as in (22).

The proof is very long and technical and we report all its details in Appendix. The expressions (20), (21), (24), and (25) are the Frechet derivatives of the functional $I$ in variables $R, m$, and $a^{\prime}$. The derivative $I_{m}{ }^{\prime}(t)$ has different forms (21) and (24) depending on whether the restriction (13) is active or inactive. Before giving the economic interpretation of the optimality conditions, some technical comments are in order.

Remark 1. If (13) is active (Case A), then the state variable $a$ is determined from $m(a(t)) a^{\prime}(t)=m(t)-E_{\max }{ }^{\prime}(t)$ and the state restriction $a^{\prime} \geq 0$ on the variable $a$ in (14) is satisfied if $E_{\max }{ }^{\prime}(t) \leq 0, t \in[0, \infty)$. If the condition $E_{\max }{ }^{\prime}(t) \leq 0$ fails for some $t \in \Delta \subset[0, \infty)$, then Theorem 1 is still valid in Case A if we replace the differential constraint $a^{\prime}(t) \geq 0$ in (14) with the stricter constraint $m(t) \geq \max \left\{0, E_{\max }{ }^{\prime}(t)\right\}$ on the control $m$ (see Hritonenko and Yatsenko, 2006, for a proof).

Remark 2. To keep mathematical complexity reasonable, we have not included the constraint $c(t) \geq 0$ into the NCE. To be complete, Theorem 1 needs to include two more cases: $E^{*}<E_{\max }$, $c^{*}=0$, and $E^{*}=E_{\max }, c^{*}=0$. The problem (10)-(17) in these cases should be treated as an OP with state constraints, which leads to certain mathematical challenges (see Hartl, Sethi, and Vickson, 1995 , for an insight into this issue). As we shall see, the regime $c^{*}(t)=0$ does not appear in the long-term dynamics (Section 4). We return to its analysis in Section 5, where it arises during the transition dynamics as one of possible scenarios. 
Remark 3. Sufficient conditions for an extremum for such OPs are complicated and involve the second Freshet derivatives of the functional $I$. The authors derived and analyzed such a condition in the form $\left|\begin{array}{ll}I_{R R}^{\prime \prime}(t) & I_{R m}^{\prime \prime}(t) \\ I_{m R}^{\prime \prime}(t) & I_{m m}^{\prime \prime}(t)\end{array}\right|<0$ at $R=R^{*}, m=m^{*}$ for Case (A). It is not included into this paper.

Remark 4. The vintage models with endogenous TC are multi-extremal under natural conditions, see Chapter 6 in Hritonenko and Yatsenko (1996). We can show that the OP (10)-(17) may also possess two local extrema:

(1) the trivial solution $R^{0}(t) \equiv 0, m^{0}(t) \equiv 0, a_{0} \leq a^{0}(t) \leq 0, t \in[0, \infty)$. This solution is verified by its substitution into (20), (24), (25), then $I_{R}{ }^{\prime}(t)<0, I_{m}{ }^{\prime}(t)<0$, and $I_{a^{\prime}}{ }^{\prime}(t)<0$, i.e., the NCE (23) holds. This local solution describes economic dynamics with no investment to technological renovation when the entire profit goes to the consumption goods. The solution is not stable in the sense that some (small) positive investments in new capital and $\mathrm{R} \& \mathrm{D}$ can force the economic system to jump to the next solution.

(2) the non-trivial solution, where $R^{*}(t), m^{*}(t), a^{*}(t)$ are positive, at least, on some parts of the planning horizon $[0, \infty)$. It describes the case where the economic system installs new equipment and invests into science and technology.

The paper focuses on the structure of the non-trivial solution $\left(R^{*}, m^{*}, a^{*}\right)$.

Let us move now to some economic interpretations of the obtained first-order optimality conditions. In order to compare more easily with the existing literature, we start with Case (A), that is, when the quota constraint is binding. Indeed, in such a case, the latter can be broadly viewed as an "equilibrium" condition in the resource market, where the quota plays the role of supply. Let us interpret the optimality conditions with respect to investment and $\mathrm{R} \& \mathrm{D}$, the case of scrapping being trivially fixed by Remark 1 above. Using equations (19) and (21), the (interior) optimal investment rule may be rewritten as:

$$
(1-\theta) \int_{t}^{a^{-1}(t)} e^{-r \tau}\left[1-\frac{\beta(a(\tau))}{\beta(t)}\right] d \tau=e^{-r t} k(t) .
$$

The interpretation of such a rule is quite natural having in mind the early vintage capital literature (notably, Solow et al., 1966, and Malcomson, 1975) as exploited in Boucekkine, Germain, and Licandro (1997). In our model, one unit of capital at date $t$ costs $k(t)$ or $e^{-r t} k(t)$ in present value. This is the right-hand side of the optimal rule 
above. The left-hand side should, therefore, give us the marginal benefit from investing. Effectively, it is the integral of discounted gains from investing over the lifetime of a machine bought at $t$ (since $a^{-1}(t)$ is by construction the lifetime of such a machine). At any date comprised between $t$ and $a^{-1}(t)$, a machine bought at $t$ will provide one unit of output but the firm has to pay the corresponding energy expenditures $\frac{\beta(a(\tau))}{\beta(t)}$. Given our Leontief specifications, $\frac{1}{\beta(t)}$ is the resource requirement of any machine bought at date $t$. Therefore, $\beta(a(\tau))$ plays the role of the effective price of the input paid by the firm. How could this be rationalized? Simply by noticing that under a binding quota, the latter mimics a clearing market condition as in the early vintage macroeconomic literature (see for example, Solow et al., 1966). ${ }^{6}$ In such a framework, the marginal productivities of energy should be equalized across vintages, implying a tight connection between the effective price of resource and the resource requirement of the oldest machine still operated. More precisely, the latter price, which happens to be the Lagrange multiplier associated to the binding environmental constraint, is equal to the inverse of the resource requirement of the oldest machine still in use, which is equal to $\beta(a(\tau))$ at any date $\tau$ comprised between $t$ and $a^{-1}(t)$. Notice that in such a case, the effective price of resource $\beta(a(\tau))$ is not generally equal to $p(t)$. The latter does not play any role since resource consumption becomes predetermined equal to $p(t) E_{\max }(t)$ in the constrained regime.

Things are completely different in the case where the quota is not binding (case B of Theorem 1). In such a case, the optimal investment rule becomes (following equation (24)):

$$
\int_{t}^{a^{-1}(t)} e^{-r \tau}\left[1-\frac{p(t)}{(1-\theta) \beta(t)}\right] d \tau=e^{-r t} k(t),
$$

and $(1-\theta) \beta(a(\tau))=p(t)$ as in the firm problem studied by Malcomson (1975), making a clear difference with respect to the constraint case A. Our framework thus extends significantly the benchmark theory to allow for situations in which resource input markets do not necessarily clear due to institutional constraints.

\footnotetext{
${ }^{6}$ In Solow et al., the role of resource is played by labor.
} 
Let us interpret now the R\&D optimal rule, which is also new in the literature. Using (20), it is given by

$$
b n R^{n-1}(t) \int_{t}^{\infty} \beta^{1-d}(\tau) m(\tau)\left[\frac{e^{-r \tau}-e^{-r a^{-1}(\tau)}}{r}(1-\theta)-e^{-r \tau} k(\tau)\right] d \tau=e^{-r t}
$$

The right-hand side is simply the present value of marginal investment in $R \& D$. The marginal benefit is given by the left-hand side. Contrary to the optimal investment rule, the gains from doing $R \& D$ last forever: the $R \& D$ investment induces a knowledge accumulation process, which is not subjected to obsolescence in our case, in contrast to capital goods. The integrand can be understood if one has in mind the maximized function (10) in the form

$$
I=\int_{0}^{\infty} e^{-r t}\left[(1-\theta) \int_{a(t)}^{t} \beta(\tau) m(\tau) d \tau-p(t) \int_{a(t)}^{t} m(\tau) d \tau-R(t)-k(t) \beta(t) m(t)\right] d t
$$

and the given endogenous law (16),(17) of motion of technological progress. It should be noticed that rewriting the problem in terms of $m(t)$, rather than in terms of investment in physical units $\mu(t)$, does not mean rewriting a problem with input-saving technical progress as a problem with output-augmenting technical progress. As one can see, at the fixed $m(t)$, an increase of $R(t)$ (and, therefore, $\beta(t)$ ) increases not only the output $Q(t)$ but also the investment expenditures through the term $k(t) \beta(t) m(t)$. The left-hand side of the optimal $R \& D$ rule takes precisely into account this trade-off. On one hand, the marginal increase in $\beta(\tau), \tau \geq t$, following the marginal rise in $R(t)$, that is, $\frac{b n R^{n-1}(t)}{\beta^{d}(\tau)}$, impacts positively output by improving the efficiency of all vintages after the date $t$. Let us notice that, since machines have a finite lifetime, this effect should be computed between $\tau$ and $a^{-1}(\tau)$ for each vintage $\tau$, which explains the factor $\frac{e^{-r \tau}-e^{-r a^{-1}(\tau)}}{r}=\int_{\tau}^{a^{-1}(\tau)} e^{-r s} d s$ in the integrand. On the other hand, the rising $\beta(t)$ increases investment expenditures (for a fixed $m(t)$ ), which explains the negative term, $e^{-r \tau} k(\tau)$, in the integrand.

Let us now move to the study of the system of the optimality conditions extracted above. We first start by seeking for exponential solutions (for naturally growing variables like 
$R(t))$, the so-called balanced growth paths (Section 4), in order to address in a standard way the critical issue of sustainable growth under constraint, which one of the main questions asked in this paper. We then move to short-term dynamics (Section 5) to identify the principal modernization routes.

\section{Analysis of optimal long-term dynamics.}

For the sake of clarity, we restrict ourselves to the case

$$
k(t)=k=\text { const }>0, \quad E_{\max }(t)=\bar{E}=\text { const }>0 .
$$

Alternative trajectories for the exogenous variables $k(t)$ and $E_{\max }(t)$ are studied in Boucekkine, Hritonenko, and Yatsenko (2008). The optimal long-term dynamics of the OP can involve interior regimes such that $I_{R}{ }^{\prime} \equiv 0$ and $I_{m}{ }^{\prime} \equiv 0$. Let us assume that the quota is active in the long run: $E(t)=E_{\max }(t)$ at $t \in\left[t_{l}, \infty\right), t_{l} \geq 0$. We will study the alternative case later. The corresponding long-term interior regime $\left(R_{\Lambda}, m_{\Lambda}, a_{\Lambda}\right)$ is determined by the system of three nonlinear integral equations

$$
\begin{gathered}
I_{R}{ }^{\prime}(t)=0, \quad I_{m}{ }^{\prime}(t)=0, \\
\int_{a(t)}^{t} m(\tau) d \tau=E_{\max }, \quad t \in\left[t_{l}, \infty\right),
\end{gathered}
$$

where $I_{R}{ }^{\prime}(t)$ and $I_{m}{ }^{\prime}(t)$ are determined by (20) and (21). The equations (27) lead to

$$
\begin{gathered}
b n R^{n-1}(t) \int_{t}^{\infty}\left[b d \int_{0}^{\tau} R^{n}(\xi) d \xi+B^{d}\right]^{1 / d-1} m(\tau)\left[\frac{e^{-r \tau}-e^{-r a^{-1}(\tau)}}{r}(1-\theta)-e^{-r \tau} k(\tau)\right] d \tau=e^{-r t}, \\
(1-\theta) \int_{t}^{a^{-1}(t)}\left[1-\left[b d \int_{0}^{a(\tau)} R^{n}(\xi) d \xi+B^{d}\right]^{1 / d} /\left[b d \int_{0}^{t} R^{n}(\xi) d \xi+B^{d}\right]^{1 / d}\right] e^{-r \tau} d \tau=k e^{-r t}
\end{gathered}
$$

at $t \in\left[t_{l}, \infty\right)$.

We will explore the possibility of exponential solutions for $R(t)$, while $m(t)$ and $t-a(t)$ are constant, to the system (28)-(30) separately in the cases $n=d, n>d$ and $n<d$. First of all, we start with the following preliminary result: if $R(t)$ is exponential, then $\beta(t)$ is almost exponential and practically undistinguishable from an exponent at large $t$ in the sense of the following lemma: 
Lemma 1. If $R(t)=R_{0} e^{C t}$ for some $\mathrm{C}>0$, then $^{7}$

$$
\beta(t) \approx R_{0}{ }^{n / d}\left(\frac{b d}{C n}\right)^{1 / d} e^{C n t / d}
$$

at large t. In particular, $\beta(t)=R_{0}{ }^{n / d}(b d / C n)^{1 / d} e^{C n t / d}$ if $b d R_{0}{ }^{n}=C n B^{d}$.

Proof. At $R(t)=R_{0} e^{C t}, \quad \beta(t)=\left(d \int_{0}^{t} b R_{0}{ }^{n} e^{C n v} d v+B^{d}\right)^{1 / d}=\left(\frac{b d R_{0}{ }^{n}}{C n} e^{C n t}-\frac{b d R_{0}{ }^{n}}{C n}+B^{d}\right)^{1 / d}$.

Dividing $\beta(t)$ by $\widetilde{\beta}(t)=R_{0}{ }^{n / d}\left(\frac{b d}{C n}\right)^{1 / d} e^{C n t / d}$, we obtain

$$
\frac{\beta(t)}{\widetilde{\beta}(t)}=\frac{1}{(b d / C n)^{1 / d} e^{C n t / d}}\left(\frac{b d}{C n} e^{C n t}+\frac{B^{d}}{R_{0}{ }^{n}}-\frac{b d}{C n}\right)^{1 / d}=\left[1+\left(\frac{C n B^{d}}{b d R_{0}{ }^{n}}-1\right) e^{-C n t}\right]^{1 / d}
$$

Expanding the function $(1+x)^{c}$ in (32) into the series, we obtain $\frac{\beta(t)}{\widetilde{\beta}(t)}=1+\varepsilon(t)$, where the small parameter $\quad \varepsilon(t)=\frac{1}{d}\left(\frac{C n B^{d}}{b d R_{0}{ }^{n}}-1\right) e^{-C n t}+\frac{1}{2 d}\left(\frac{1}{d}-1\right)\left(\frac{C n B^{d}}{b d R_{0}{ }^{n}}-1\right)^{2} e^{-2 C n t}+\ldots$ decreases as $e^{-C n t}$. The lemma is proved.

We now define the concept of balanced growth paths considered.

Definition 1. The Balanced Growth Path (BGP) is a solution (R, $m, a)$ to (28), (29) and (30), where $R(t)$ is exponential and $m(t)$ and $t-a(t)$ are positive constants, which satisfy constraints (14), in particular, the non-negative cash-flow requirement.

If the quota is not binding, then the system to be solved is

$$
I_{R}{ }^{\prime}(t)=0, \quad I_{m}{ }^{\prime}(t)=0, \quad I_{a^{\prime}}(t)=0, \quad t \in\left[t_{l}, \infty\right),
$$

where $I_{R}{ }^{\prime}(t), I_{m}{ }^{\prime}(t)$ and $I_{a^{\prime}}(t)$ are determined by (20), (24), and (25). As shown below, the optimal long-term growth with inactive regulation, $E<E_{\max }$, is possible only at $n>d$ (see Section 4.2).

Remark 5. In the case of the inactive quota constraint, it is convenient to introduce the Frechet derivative

\footnotetext{
${ }^{7}$ For brevity, we will omit the expression "at large $t$ " when using the notation $f(t) \approx g(t)$
} 


$$
I_{a}{ }^{\prime}(t)=(1-\theta) e^{-r t}\left[\bar{P} e^{\gamma t}-\beta(a(t))\right] m(a(t))
$$

in $a$ instead of the derivative (25) in $a^{\prime}$ and use it during the BGP analysis. Indeed, it is easy to see that if $I_{a^{\prime}}(t) \equiv 0$ at $t \in\left[t_{l}, \infty\right)$ for some $t_{l} \geq 0$, then $I_{a}{ }^{\prime}(t) \equiv 0$ at $t \in\left[t_{l}, \infty\right)$.

\subsection{Balanced growth in case $n=d$.}

Let the parameter $n$ of "R\&D efficiency", $0<n<1$, be equal to the parameter $d$ of "R\&D complexity", $0<d<1$. In this case, the optimal long-term growth involves the active regulation at natural conditions. More precisely, we get the following important characterization:

Lemma 2. At $n=d$, any interior solution ( $R, m$, a) of the $O P$ (10)-(17) with an exponentially growing $R(t)$ involves the binding quota $E(t)=E_{\max }$ starting at some $t_{k} \geq 0$, under the condition $\gamma<C$, where $C$ is the endogenous rate of optimal $R(t)$.

Proof. Let us consider $R(t)=R_{0} e^{C t}$, then $\beta(t) \approx R_{0}(b / C)^{1 / d} e^{C t}$ by Lemma 1 .

We assume that $E(t)<E_{\max }$ at $\left[t_{l}, \infty\right), t_{\geq} \geq 0$. Then, by Theorem 1 , an OP interior regime $(R$, $m, a$ ) has to satisfy the nonlinear system (33) on $\left[t_{l}, \infty\right)$. Substituting the above $R$ and $\beta$ into the expressions (20) and (34) for $I_{R}{ }^{\prime}(t)$ and $I_{a}{ }^{\prime}(t)$, we obtain from (33) that

$$
\begin{gathered}
R_{0}(b / C)^{1 / d} e^{C a(t)}=\bar{P} e^{\gamma t}, \\
b d(b / C)^{1-d} e^{C(d-1) t} \int_{t}^{\infty} e^{C(1-d) \tau} e^{-r \tau}\left[\frac{1-e^{-r a^{-1}(\tau)}}{r}(1-\theta)-k\right] m(\tau) d \tau \approx e^{-r t}, \quad t \in\left[t_{l}, \infty\right) .
\end{gathered}
$$

Equation (35) determines $a$, which is such that $t-a(t) \rightarrow \infty$ at $t \rightarrow \infty$ because of $\gamma<C$. Equation (36) determines $m$ at a given $a$. After introducing the function $f(t)=$ $(1-\theta)\left[1-e^{-r\left[a^{-1}(t)-t\right]}\right] / r-k$ and differentiating (36), we have

$$
m(t)=[r-C(1-d)](C / b)^{1-d} / b d / f(t) .
$$

Since $f(t)<(1-\theta) / r$ for any possible $k$ and $a$, then $m(t)>$ const $=r[r-C(1-d)](C / b)^{1-d} / b d /(1-\theta) / r>0$. Therefore, by (13), $E(t)$ increases indefinitely at $t \rightarrow \infty$, our assumption is wrong and $E(t)=E_{\max }$ at some $t_{k}>t_{l}$. The lemma is proved. 
Some comments are in order here. First of all, though it involves a control variable, that is, $R$ at the minute, the restriction $\gamma<C$ on the price of energy is still highly interesting. Of course, it is important to observe that since the growth rate $C$ is endogenous, it may depend on $\gamma$, and, therefore, the restriction might be impossible. We will show that it is not whence the optimal control $R(t)$ better characterized and the optimal growth rate $C$ uncovered. Nonetheless, the restriction $\gamma<C$ sounds as a natural (sufficient) condition for the firm to overcome increasing scarcity as reflected by a strictly positive growth rate of the energy price. The fact that a permanent growth regime does imply active regulation at a finite time is much less surprising, provided such a regime exists. Second, the restriction $\gamma<C$ is indeed sufficient. We will see below that a balanced growth with the active quota regulation can take place at $\gamma=C$ as well. This is part of the following key theorem which essentially establishes the existence of balanced growth paths in the sense of Definition 1 when the economic and institutional environments are held constant. This theorem is crucial in that it fully characterizes the endogenous growth rate, $C$.

Theorem 2 (about the balanced growth). At $n=d$ and $\gamma \leq C$, the interior optimal regime $-B G P\left(R_{\Lambda}, m_{\Lambda}, a_{\Lambda}\right)$ exists,

$$
R_{\Lambda}(t) \approx \bar{R} e^{C t}, \quad Q_{\Lambda}(t), \beta_{\Lambda}(t), c_{\Lambda}(t) \sim e^{C t}, \quad m_{\Lambda}(t)=\bar{M}=\mathrm{const}, \quad a_{\Lambda}(t)=t-\bar{E} / \bar{M},
$$

where the constants $C$ and $\bar{M}$ are determined by the nonlinear system

$$
\begin{gathered}
C^{1 / d}[r / C+d-1]=d b^{1 / d} \bar{M}\left[\frac{1-e^{-r \bar{E} / \bar{M}}}{r}-\frac{k}{1-\theta}\right](1-\theta), \\
\frac{1-e^{-r \bar{E} / \bar{M}}}{r}-\frac{e^{-C \bar{E} / \bar{M}}-e^{-r \bar{E} / \bar{M}}}{r-C}=\frac{k}{1-\theta},
\end{gathered}
$$

that has a positive solution, at least, at small $r$. Namely, if $r<<1$ and

$$
r^{1 / d}<\bar{E} b^{1 / d}[1-\sqrt{2 k r /(1-\theta)}],
$$

then $C, 0<C<r$, is a solution of the nonlinear equation

$$
C^{(1-d) / d}[r-C(1-d)]=d \bar{E} b^{1 / d}\left[1-\sqrt{\frac{k}{2(1-\theta)}}\left(\frac{r}{\sqrt{C}}+\sqrt{C}\right)\right]+o(r)
$$

and $\bar{M}=\bar{E} \sqrt{C / 2 k /(1-\theta)}+o(r)$. Therefore, the growth rate $C$ does not depend on the energy price but does depend on the regulation parameters $\bar{E}$ and $\theta$. 
The uniqueness of the solution is guaranteed if

$$
r^{1 / d-1 / 2}<\frac{d^{2} b^{1 / d} \bar{E}}{4(1-d)} \sqrt{\frac{2 k}{1-\theta}} .
$$

If $\gamma<C$, then the BGP (38) exists for any $\bar{R}>0$. If $\gamma=C$, then the BGP (38) is possible at the condition

$$
\bar{R}>\bar{E} \bar{P} \frac{C d}{(r-C)(1-\theta)}
$$

The proof is long and it is reported in Appendix. Before commenting on the theorem, let is state the following useful corollary.

Corollary 1. For a given energy price growth rate $\gamma$, let $C$ be the solution of (39)-(40), $C$ be independent of $\gamma$, then, no BGP is possible at $\gamma>C$.

The proof immediately follows from the proof of Theorem 2 provided in Appendix (precisely, from formula (A13): indeed, if $\gamma>C$, then $c_{\Lambda}(t)<0$ at large $t$ ).

Theorem 2 and its Corollary make clear when the firm can still grow in the long-run despite scarcity. Without scarcity $(\gamma=0)$, sustainable growth regimes are possible despite input quotas and liquidity constraints. And such a property actually holds up to a certain threshold level of the growth rate of resource price (or of the scarcity degree). Theorem 2 brings more striking results. First of all, it should be noted that the resource price $p(t)=\bar{P} e^{\imath t}$ is not involved in the BGP (38) when $₹<$. In particular, the overall innovation rate is independent of the characteristics of the energy price patterns. In our model, this property comes naturally: if $\mathcal{K} C$, then by Lemma 2 any balanced growth regime should involve active regulation (or a binding quota), and active regulation eliminates the resource price from the NCE formulas (18)-(22), as stated and economically interpreted in Section 3. So, the optimal long term dynamics $\left(R_{\Lambda}, m_{\Lambda}, a_{\Lambda}\right)$ are the same for any resource price path up to a certain rate. Second, this important property does not mean that energy prices play no role in the long-run. On one hand, Corollary 1 shows clearly that there is no hope to have a BGP if the growth rate of resource price (or in other words the degree of scarcity) is large enough. We will show 
clearly in Section 5.1 that in such a case, the firm collapses. On the other hand, the resource price does matter in the edge-of-knife case $\gamma=C$. Indeed, in the latter case, while the price level $\bar{P}$ still does not impact the endogenous balanced economy rate $C$, the cash-flow non-negativity requirement precisely implies condition (44), which limits the long-run R\&D investment level depending on the value of $\bar{P}$.

Finally, notice that even if $\gamma<C$, and while the long-term optimal policy is to invest the same in machines and in $R \& D$ whatever the resource price trajectory is, the latter does affect the optimal value of functional (10), since it impacts the optimal cash flows $c^{*}$ : higher $p$ means a lower level of cash $c$. We shall see in Section 5 that the resource price impacts crucially the transition dynamics in our model, and not only via the collapse cases mentioned just above. The role of this price in the long-run dynamics is also a valid question when $n>d$ (then the environmental regulation is not binding) and will be considered in Section 4.2 hereafter.

Some more technical comments on comparative statics are useful. First of all, more can be said about the uniqueness of the growth rates $C$ compatible with the BGP requirements. Indeed, if equation (42) has a solution $0<C<r$, then, in the general case, it has another solution $C_{2}, r<C_{2}<r /(1-d)$. However, the larger solution $C_{2}$ has no sense, since at $C>r$ the value of (1) is infinite and $c^{*}(t)<0$ (by (A13) given in Appendix). Second, it is very important to notice that in Theorem 2, the BGP scale parameter $\bar{R}$ is actually undetermined. We have the indeterminacy of the long-term dynamics under the BGP, because technical progress is endogenous. It happens for similar problems in the endogenous growth theory. A typical example is the Lucas-Uzawa model (Boucekkine and Ruiz-Tamarit, 2008).

We shall now move to comparative statics, which are particularly useful in the context of Theorem 2 and its Corollary. Since the BGP growth rate $C$ is independent of $\gamma$, what are the possibilities to alter the value of $C$, for a given, possibly higher energy price growth rate? The needed properties are summarized hereafter.

Corollary 2. At (26) and $r<<1$, a decrease of $\bar{E}$ leads to the decrease of both optimal parameters $C$ and $\bar{M}$, and leaves the long-term lifetime of capital goods unaltered since 
$\bar{M} \sim \bar{E}$. A decrease of capital price $k$ and/or of the $\operatorname{tax} \theta$ increases the optimal $C$ and $\bar{M}$ and diminishes the long-term lifetime of capital goods.

More stringent regulation through a decrease in $\bar{E}$ is bad for the growth rate of firms' output and profit. Even though the firms can respond to tighter quotas by more innovation, such an instrument does not allow to completely circumvent the impact of more severe regulation. Lower capital prices are good for investment (in resource consumptions units) and also prove to be beneficial for the growth rate of firms' output and profit. Lower equipment prices make firms wealthier and such a positive wealth effect boost the investment either in capital or in R\&D. For the same reasons, a lower tax rate $\theta$ raises the optimal growth rate, $C$, and stimulates the two latter forms of investment. It should be noted at this point that both regulation parameters $\bar{E}$ and $\theta$ do matter in the BGP growth rate $C$. And they work in a way opposite to the Porter hypothesis, depressing growth and investment in the long-run.

A further interesting result concerns the optimal long-term lifetime of capital goods. Since $a_{\Lambda}(t)=t-\bar{M} / \bar{E}$, and $\bar{M} \sim \bar{E}$, it follows that a tighter environmental regulation leaves the optimal lifetime of capital goods unaltered. While a lower $\bar{E}$ does reduce the optimal lifetime of machines, such a tighter regulation also pushes the investment downward, which forces the maximizing firm to use fewer machines longer. These two effects appear to offset each other in our framework. Under decreasing prices for capital goods (or a falling tax rate), the firm invests more and uses the machines for a shorter time. This is somehow consistent with the recent literature on embodied technological progress observing that a more rapid investment-specific technological progress (like the one conveyed by the information and communication technologies) reduces the relative price of capital goods and decreases their lifetime due to rising obsolescence costs (see, for example, Krusell, 1998).

\subsection{Cases $n<d$ and $n>d$.}

In these cases, no BGP in the sense of Definition 1 exists. However, a long-term regime with exponentially growing $R$ and decreasing $m$ appears to be possible at a special 
combination of given parameters (see also Yatsenko, Boucekkine and Hritonenko, 2009, for other related dynamics)

Theorem 3. Let (26) hold. Then:

(a) If $n<d$, then no interior optimal regime with an exponentially growing $R$ exists.

(b) If $n>d$, then an interior optimal regime $\left(R_{\Lambda}, m_{\Lambda}, a_{\Lambda}\right)$ such that $R_{\Lambda}(t)$ grows exponentially, $m_{\Lambda}(t) \rightarrow 0$ at $t \rightarrow \infty$, and $E(t)<E_{\max }$, is possible ONLY if $\gamma=C$, where $C$ is the endogenous rate of $R_{\Lambda}(t)$.

The proof is provided in Boucekkine, Hritonenko, and Yatsenko (2008). When $n>d$, the efficiency of the R\&D investment appears to be higher as compared with the investment into the new capital. Theorem 3 concludes that, in the optimal long-time regime, almost all the output goes to R\&D investment and the part of capital investments (exponentially) decreases in the total distribution of the output. Also, the quota constraint needs not to be binding and we can keep a larger amount of older assets (since we buy an increasingly smaller amount of new capital).

In addition, by (29), the restriction $k<(1-\theta) / r$ on the given capital price is necessary for the existence of any positive optimal regime. The resource price $\bar{P} e^{\gamma t}$ plays a decisive role in the case $n>d$, in particular, an interior optimal path with an exponential $R_{\Lambda}$ is impossible if $\gamma=0$ (the resource price does not increase). Only when the resource price increases at the rate $\gamma=C$, an interior regime with exponentially increasing $R_{\Lambda}$ and decreasing $m_{\Lambda}$ is possible. The increase of $\bar{P} e^{\varkappa}$ raises $a_{\Lambda}(t)$, that is, decreases the lifetime of capital goods. In other words, a kind of induced-innovation mechanism seems to be active in the case $n>d$, that is, when the R\&D activity is highly efficient, so efficient that the investment into equipment goes to zero. In such a case, the firm is in perpetual sharp modernization, and is not suffering at all from any regulation. We have to notice that this regime is not a BGP in the sense of Definition 1, because $m_{\Lambda}(t)$ asymptotically tends to zero. We shall disregard such a configuration in the short-term dynamics section below.

\section{Transition dynamics: Collapse cases and optimal modernization strategies}


From now on, we set $n=d$. For simplicity sake, let assumptions (26) hold.

Since we have to deal with short-term dynamics in this section, some comments on initial conditions are in order. The OP solution $\left(R^{*}, m^{*}, a^{*}\right)$ satisfies the initial conditions (15). An essential initial condition is $a(0)=a_{0}$ because the endogenous $a(t)$ is continuous. If $a_{0} \neq a_{\Lambda}(0)$, then the dynamics of $\left(R^{*}, m^{*}, a^{*}\right)$ involves a transition from the initial state $a(0)=a_{0}$ to the long term interior trajectory $a_{\Lambda}$.

By $(14), c(0)=(1-\theta) Q(0)-p(0) E(0)-R(0)-k \beta(0) m_{0}(0) \geq 0$ at the initial state $t=0$, or

$$
\bar{P} E(0)+k B m_{0}(0) \leq(1-\theta) \int_{a_{0}}^{0}\left[\beta_{0}+\int_{a_{0}}^{\tau} R_{0}(v) d v\right] m_{0}(\tau) d \tau-R_{0}(0) .
$$

Otherwise, the economic system is not possible at $t=0$ because the resource and capital prices $p(0)=\bar{P}$ and $k$ are too high. Condition (45) implies two simpler constraints:

$$
\bar{P}<(1-\theta) B \quad \text { and } \quad k m_{0}(0)<E(0)(1-\theta) .
$$

Even if (45) holds, the optimal dynamics may be such that the economic system will never reach the restriction $E(t)=E_{\max }$ because of high resource and/or capital prices. Let us demonstrate the corresponding scenarios.

\subsection{The collapse cases.}

Let $E(0)=\int_{a_{0}}^{0} m_{0}(\tau) d \tau<E_{\max }$ at the initial time $t=0$.

Scenario 1: The case of too high resource price. Let us assume that the market resource price $p(t)=\bar{P} e^{\gamma t}$ increases faster than the optimal $\beta(t): \gamma>C$. Then, by (25),

$$
I_{a^{\prime}}{ }^{\prime}(t)=\int_{t}^{\infty} e^{-r \tau}\left[\bar{P} e^{\gamma \tau}-(1-\theta) \beta(a(\tau))\right] m(a(\tau)) d \tau,
$$

$I_{a^{\prime}}{ }^{\prime}(t)>0$ for all $t$, and the optimal strategy is to keep the lifetime of the capital $t-a^{*}(t)$ as short as possible because of the high resource cost $\bar{P} e^{\gamma}$. In this case, the optimal $a^{*}(t)$ soon becomes $a^{*}(t)=t$ and the optimal investment $m^{*}(t)=0$ is determined by the sign $I_{m}{ }^{\prime}(t)<0$ in

$$
I_{m}^{\prime}(t)=\int_{t}^{a^{-1}(t)} e^{-r \tau}\left[(1-\theta) \beta(t)-\bar{P} e^{\gamma \tau}\right] d \tau-e^{-r t} k \beta(t) .
$$


Finally, by (20),

$$
I_{R}{ }^{\prime}(t)=b n R^{n-1}(t) \int_{t}^{\infty} \beta^{1-d}(\tau) m(\tau)\left[\frac{e^{-r \tau}-e^{-r a^{-1}(\tau)}}{r}(1-\theta)-e^{-r \tau} k\right] d \tau-e^{-r t},
$$

therefore $I_{R}{ }^{\prime}(t)<0$ implying $R^{*}(t)=0$. So, the optimal dynamics reflects a situation of economic collapse (no capital renovation and complete scrapping of existing capital) because of too high price of the resource. The variable $E(t)=\int_{a(t)}^{t} m(\tau) d \tau$ strives to 0 and is always less than $E_{\max }$.

Scenario 2: The case of too high capital cost k (or too high tax $\theta$ ). Let the market price $k$ of new capital be $k \geq(1-\theta) / r$. Then, by (48), $I_{m}{ }^{\prime}(t)<0$ at $t>0$ and the optimal new investment $m^{*} \equiv 0$. So, the optimal investment strategy is to buy no new capital. By (49), $I_{R}{ }^{\prime}(t)<0$ and the optimal $R^{*} \equiv 0$. By (47), if $\gamma=0$ (the resource price does not increase), then $I_{a^{\prime}}(t)<0$ and, hence, $a^{*} \equiv a_{0}$. If $\gamma>0$, then $a^{*}(t)$ may also increase. Regardless of that, we have $E(t)=\int_{a(t)}^{0} m_{0}(\tau) d \tau \leq E(0)<E_{\max }$, meaning that the quota constraint is never binding. The optimal dynamics feature an economic decline (no R\&D investment, no capital renovation and no capital scrapping) because of too high price $k$ of new capital. The same situation $k \geq(1-\theta) / r$ happens if the tax $\theta$ is too high.

The above scenarios do not enhance the nature of technological capital replacement. Below, we consider cases when the optimal system dynamics involves capital renovation and an optimal modernization process takes place.

The OP produces qualitatively different optimal regimes $\left(R^{*}, m^{*}, a^{*}\right)$ depending on whether the quota restriction (13) is active, $E(t)=E_{\max }$, or inactive, $E(t)<E_{\max }$. If the resource is energy, the firms-polluters are the firms for which (13) is active. We will consider the cases of firms-polluters and firms-non-polluters separately, under the conditions $\gamma<C$ and $k<(1-\theta) / r$ to rule out the collapse cases mentioned above. By Theorem 3, the long-term BGP dynamics involves the active quota restriction (13). As shown below, the transition dynamics reach the restriction (13). 


\subsection{Optimal intensive growth (the case of a dirty firm).}

Let us assume that $E(t)=E_{\max }$ starting with an instant $t_{\mathrm{k}}, t_{\mathrm{k}} \geq 0$.

Scenario 3: The intensive growth at the active resource quota. Let $t_{\mathrm{k}}=0$. The optimal dynamics at $t \geq t_{\mathrm{k}}$ follows Case $\mathrm{A}$ of Theorem 1 (with the $E(t)=E_{\max }$ restriction). This regime is a growth with intensive capital renovation induced by technical progress. In order to make a new capital investment $m(t)$, the firm needs to scrap some obsolete capital $m(a(t)) a^{\prime}(t)$, following equality (13) or

$$
\int_{a(t)}^{t} m(\tau) d \tau=E_{\max }
$$

In the long-term dynamics considered in Section 5.1, the optimal R\&D innovation $R^{*}(t)$ is the interior trajectory $R_{\Lambda}(t)$ determined from $I_{R}{ }^{\prime}(t)=0$, where $I_{R}^{\prime}(t)$ is given by (49). The optimal $R^{*}(t)$ reaches the trajectory $R_{\Lambda}(t)$ immediately after $t_{\mathrm{k}}$. The OP has the interior turnpike trajectory $a_{\Lambda}$ for the capital lifetime, determined from $I_{m}{ }^{\prime}(t)=0$ or

$$
\int_{t}^{a^{-1}(t)} e^{-r \tau}(1-\theta)[\beta(t)-\beta(a(\tau))] d \tau=e^{-r t} k \beta(t) .
$$

If $a_{\Lambda}(0)=a_{0}$, then the optimal $a^{*} \equiv a_{\Lambda}$. If $a_{\Lambda}(0) \neq a_{0}$, then we can show that the optimal $a^{*}(t)$ will reach $a_{\Lambda}(t)$ at some time $t_{\mathrm{P}}>t_{\mathrm{k}}$. If $a_{\Lambda}(0)<a_{0}$, then the optimal investment $m^{*}(t)=0$ is minimal at $0<t \leq t_{1}$ (otherwise, $c^{*}(t)=0$ and $m^{*}(t)$ is determined by additional considerations). Later, at $t>t_{1}$, the optimal $m^{*}(t)$ has cycles (the replacement echoes as in Boucekkine, Germain and Licandro, 1997) determined by the prehistory of $m(t)$ on $\left[a_{0}\right.$, $t_{\mathrm{k}}$ ]. A formal proof of this optimal $m^{*}, a^{*}$ dynamics can be done similarly to Hritonenko and Yatsenko (2005).

Under Scenario 3, the resource price does not enter the NCE formulas (18)-(22). However, an increase of the resource price reduces the corresponding optimal $c^{*}(t)$ and, therefore, can make the optimal transition longer (if $\left.a_{\Lambda}(0)>a_{0}\right)$. Figure 1 and the following simulation example illustrate this scenario.

Example 1. Let

$$
r=0.05, \quad d=n=0.5, \quad b=0.01, \quad E_{\max }(t)=\bar{E}=10.5, \quad k(t)=0.12, \quad p(t)=0.5,
$$




$$
\theta=0, \quad a_{0}=-2, \quad \beta_{0}=1, \quad R_{0}(\tau)=0, \quad m_{0}(\tau)=5.25, \quad \tau \in[-2,0] .
$$

and $B=\beta(0)=1$ by (16). Then, there is the BGP, determined by Theorem 2 above,

$$
R_{\Lambda}(t)=\bar{R} e^{C t}, C=0.01, \quad m_{\Lambda}(t)=\bar{M}=2.1, \quad a_{\Lambda}(t)=t-5 \quad, t \in[0, \infty),
$$

indicated by the grey lines in Figure 1 . In this case, $E(0)=m_{0} a_{0}=5.25^{*} 2=10.5$ is equal to $E_{\max }(0)=$ $\bar{E}$, hence, the quota (13) is active starting $t=0$. Since $a_{A}(0)=-5<a_{0}=-2$, then the optimal $a^{*}(t)=-2$ and $m^{*}(t)=0$ at $0<t \leq t_{1}=3$. After $t_{\mathrm{l}}$, the optimal $a^{*}(t)$ coincides with the BGP $a_{\Lambda}(t)$ and $m *(t)=m *(t-5)$ exhibits replacement echoes.

\subsection{Optimal extensive growth (the case of a firm-non-polluter).}

This case means that the consumed resource $E(t)$ at the initial state $t=0$ is lower than the limit $E_{\max }$. Let us assume that $E(t)<E_{\max }$ at $0 \leq t<t_{\mathrm{k}}$, where the moment $t_{\mathrm{k}}$ will be determined. Mathematically, this scenario is more complicated and involves the case $c^{*}(t)=0$, not covered by Theorem 1 .

We assume that $p(t)=\bar{P} e^{\imath t}$ is not too high, so that

$$
I_{m}{ }^{\prime}(t)=\int_{t}^{a^{-1}(t)} e^{-r \tau}\left[(1-\theta) \beta(t)-\bar{P} e^{\gamma \tau}\right] d \tau-e^{-r t} k \beta(t)>0
$$

on the "extensive-growth" part $\left[0, t_{\mathrm{k}}\right]$ of transition dynamics (the alternative case of too high $p(t)$ is Scenario 1 above). Then, $m^{*}(t)$ is maximal on interval $\left[0, t_{\mathrm{k}}\right]$. On the other side, by (49), $I_{R}{ }^{\prime}(t)>0$ at small $R^{*}(t)$, hence, the optimal $R^{*}(t)$ is positive. In this case, the constraint $c^{*}(t) \geq 0$ in (14) limits both controls $R^{*}$ and $m^{*}$ :

$$
(1-\theta) Q^{*}(t)-R^{*}(t)-k(t) \beta^{*}(t) m^{*}(t)-p(t) E^{*}(t) \geq 0 .
$$

Then, the transition optimal dynamics on some initial period $\left[0, t_{\mathrm{k}}\right]$ is determined by the restriction $c^{*}(t)=0$, i.e., the optimal $m^{*}(t)$ and $R^{*}(t)$ are connected by

$$
R^{*}(t)+k(t) \beta^{*}(t) m^{*}(t)=(1-\theta) Q^{*}(t)-p(t) E^{*}(t), \quad t \in\left[0, t_{\mathrm{k}}\right] .
$$

Therefore, we need a first order condition for this case.

Lemma 3. If $E^{*}(t)<E_{\max }(t)$ and $c^{*}(t)=0$ at $t \in\left[0, t_{\mathrm{k}}\right]$, then the interior optimal $R^{*}(t)>0$ satisfies the condition 


$$
\widehat{I}_{R}{ }^{\prime}(t)=b n R^{n-1}(t) \int_{t}^{\infty} \beta^{1-d}(\tau) m(\tau)\left[(1-\theta) \int_{\tau}^{a^{-1}(\tau)} \chi(\xi) d \xi-\chi(\tau) k(\tau)\right] d \tau-\chi(t)=0,
$$

where the function $\chi(t)=\exp (-r t)$ at $t \in\left[t_{\mathrm{k}}, \infty\right)$ and $\chi(t)$ is found from the following Volterra integral equation of the second kind:

$$
\chi(t) k(t) \beta(t)=\int_{t}^{t_{k}} e^{-r(\tau-t)}[(1-\theta) \beta(t)-p(\tau)] \chi(t) d \tau+\int_{t_{k}}^{a^{-1}(t)} e^{-r \tau}[(1-\theta) \beta(t)-p(\tau)] d \tau
$$

at $t \in\left[0, t_{\mathrm{k}}\right]$. The optimal $m^{*}(t)>0$ is found from (53) at the given $R^{*}(t)$, and the optimal $a^{*}(t)=0$ at $I_{a^{\prime}}(t)<0$ or $a^{*}(t)>0$ satisfies $I_{a^{\prime}}(t)=0$, where

$$
I_{a^{\prime}}{ }^{\prime}(t)=\int_{t}^{\infty} e^{-r \tau}[p(\tau)-(1-\theta) \beta(a(\tau))] m(a(\tau)) d \tau \text {. }
$$

Proof is provided in Appendix.

Lemma 3 defines the following extensive-growth scenario on some initial period $\left[0, t_{\mathrm{k}}\right]$ of the transition optimal dynamics.

Scenario 4: Extensive growth. If $\gamma=0$ (the price $p(t)$ is constant), then by $(56) I_{a^{\prime}}(t)<0$ at $a \equiv a_{0}$, hence, $a^{*} \equiv a_{0}$ is optimal while $E(t)<E_{\max }$ (as in Example 2 below). So, one can buy a new capital and there is no need to remove the old one, i.e., we have an extensive growth. If $\gamma>0\left(p(t)\right.$ increases), then the optimal $a^{*}(t)=0$ while $\bar{P} e^{\gamma t}<\beta_{0}$ and $a^{*}(t)$ increases following $I_{a}{ }^{\prime}(t)=0$ at $\bar{P} e^{\gamma}>\beta_{0}$ on $\left[0, t_{\mathrm{k}}\right]$. If $\gamma<C(p(t)$ increases slower than the BGP), then the optimal capital lifetime $t-a^{*}(t)$ increases while $E(t)<E_{\max }(t)$.

By $(54), I_{R}{ }^{\prime}(t)>0$ at small $R^{*}(t)$, hence, the optimal $R^{*}(t)$ is positive and, therefore, $\beta^{*}(t)$ increases. If $\beta^{*}(t)$ increases, then by (52) the optimal investment $m^{*}$ is maximal and is determined by Lemma 3 while $E(t)<E_{\max }$. Since both optimal $m^{*}$ and $t-a^{*}(t)$ increase, the quota $E(t)=E_{\max }$ will be reached soon and the optimal renovation dynamics will switch to Scenario 3 with the active constraint (13). The end $t_{\mathrm{k}}$ of the "extensive-growth" transition period $\left[0, t_{\mathrm{k}}\right]$ is determined from condition $E\left(t_{\mathrm{k}}\right)=E_{\max }$.

Example 2. Let all given parameters be as (50) in Example 1 but

$$
m_{0}(\tau)=2, \quad \tau \in[-2,0]
$$

Then the BGP (51) is the same as in Example 1 but the transition dynamics is different. 
In this case, $E(0)=m_{0} a_{0}=2 * 2=4$ is less than $E_{\max }(0)=\bar{E}=10.5$, hence, the quota (13) is inactive on an initial interval $\left[0, t_{\mathrm{k}}\right]$ at the beginning of the planning horizon. The dynamics of optimal $m^{*}(t)$ and $R^{*}(t)$ on $\left[0, t_{\mathrm{k}}\right]$ follow the restriction $c^{*}(t)=0$ and is shown in Figure 2 . The determination of $m^{*}$ and $R^{*}$ is based on Lemma 3. It appears that $m^{*}(t)=17.8, R^{*}(t)=0.003$ at $0 \leq t \leq t_{\mathrm{k}}$. Then, the corresponding $E^{*}(t)$ increases fast and reaches the limit value $\bar{E}=10.5$ at $t_{\mathrm{k}} \approx 0.36$. The later optimal dynamics on $\left[t_{\mathrm{k}}, \infty\right)$ is described by Case A of Theorem 1 and is similar to Scenario 3 . Namely, since $a_{\Lambda}(0.36)<a_{0}=-2$, then $a^{*}(t)=-2$ and the optimal $m *(t)=0$ is minimal during the second part of transition dynamics, $0.36<t \leq t_{1}=3$. Later, at $t>3, a^{*}(t)=a_{\Lambda}(t)$ and the optimal $m *(t)=m *(t-5)$ is determined by its previous dynamics on $[-2,3]$.

The optimal dynamics highlighted in this scenario are quite new in the related economic literature (see for example, Boucekkine, Germain and Licandro, 1997). They deserve some comments:

i) At first, note that the modernization policy chosen by the firm consists of increasing investment in new equipment and R\&D without scrapping the older and more resource consuming machines. In Hritonenko and Yatsenko (1996) and Boucekkine et al. (1997), the modernization policy also encompasses scrapping part of the older capital goods in a way similar to the intensive growth scenario described in Section 5.2. The reason behind this difference is quite elementary: a firm with a low enough initial capital stock (and so, with low enough initial resource consumption) has no incentive to scrap its old machines as long as its resource quota constraint is not binding. In contrary, at a binding quota, investing in new machines is not possible without scrapping some obsolete older machines because of market clearing conditions or binding regulation constraints.

ii) Note that in our case firms which are historically "small" polluters are precisely those which are historically "small" producers. Extended to a country level, our exercise predicts that historically poor countries will find it optimal to massively invest and, therefore, to massively pollute during their development process. During such a transition, new and clean machines will co-exist with old and dirty machines in the productive sectors, implying an unambiguously dirty transition. 
We complete our analysis of optimal modernization strategies by examining explicitly how the Hicksian mechanisms work in our model.

\subsection{The impact of resource price on extensive growth.}

In the case of extensive growth (Scenario 4), the transition dynamics is directly impacted by the behavior of the resource price $\bar{P} e^{\gamma t}$ because of (52). Let $\gamma>0$ and, for simplicity sake, $\theta=0$.

Theorem 4. If the transition dynamics period $\left[0, t_{\mathrm{k}}\right]$ with $E^{*}(t)<E_{\max }$ is relatively short such that $\beta_{0} t_{k}<<1$ and $t_{k} \max _{\left[a_{0}, 0\right]} m_{0}<<1$, then an increase of the resource price $p(t)$ increases the $R \& D$ investment $R^{*}(t)$, decreases capital investment $m *(t)$, and increases the length $t_{\mathrm{k}}$ of the period. In the case of an arbitrary interval $\left[0, t_{\mathrm{k}}\right]$, the $R \& D$ investment $R *(t)$ increases, at least, on some parts of $\left[0, t_{\mathrm{k}}\right]$. The capital lifetime $t$ - $a *(t)$ remains the same while $\bar{P} e^{\imath t}<\beta_{0}$ and becomes shorter when $\bar{P} e^{\gamma t}>\beta_{0}$.

The proof is in Appendix.

Apart from technicalities, Theorem 4 allows us to deepen the knowledge on functioning of the induced-innovation Hicksian mechanisms in a sophisticated model like ours where firms have three controls in hand. The theorem establishes the existence of such a modernization mechanism, at least, during some time along the transition. It is not difficult to construct extreme cases, not covered by Theorem 4, where the Hicksian mechanism fails to appear. ${ }^{8}$ However, the general wisdom one has to extract from this exercise is that the latter mechanism is still relevant in sophisticated models like ours.

This said, the picture conveyed by Theorem 4 is an accurate and peculiar modernization process. While rising resource prices may stimulate $R \& D$ at some points in time, they definitely depress investment in capital goods. It becomes optimal for the firm to find an

\footnotetext{
8 Consider the extreme case of the prices $p_{1}(t)$ and $p_{2}(t)$ such that $(1-\theta) Q(0)-p_{1}(0) E(0)>0$ but $(1-\theta) Q(0)-p_{2}(0) E(0)=0$. The corresponding optimal $a^{*}, m^{*}$, and $R^{*}$ are indicated with the subscripts ${ }_{1}$ and ${ }_{2}$. Then, by (58), $R_{1} *(0)$ and/or $m_{1} *(0)$ are positive, but $R_{2} *(0)=m_{2} *(0)=0$ since all given output $(1-\theta) Q(0)$ is spent at $t=0$ to buy resources. Under natural assumptions, the production will never become profitable at the price $p_{2}$. There is no contradiction with Theorem 5 since the control $R_{2} *(0)=m_{2} *(0)=0$ is not interior in this case and an increase $\delta R^{*}{ }_{2}$ of $R^{*}{ }_{2}$ is not possible because of too tight initial conditions.
} 
efficient way to save resources, and to delay the investment in equipment. Modernization also works via scrapping, provided resource prices are shifted to large enough levels. In such a case, the firms start to scrap older equipment and technologies while decreasing investment in equipment and increasing the resource-saving $R \& D$ effort. This is quite consistent with available descriptions of how the inducement works in practice. For example, Newell et a. (1999) make it clear that a major observed effect of changes in energy process and in energy-efficiency standards is the commercialization of new models and elimination of old ones.

\section{Concluding remarks}

In this paper, we have studied in depth the optimal behavior of a firm subject to environmental-based quotas, resource scarcity and liquidity constraints. In addition, the vintage structure adds realism to the problem under study and considerably enriches the discussion. We have extracted some new results, either in the investigation of short-term dynamics (optimal modernization strategies) or in the analysis of long-run growth regimes (sustainability). We have also characterized the general contours of the Hicksian mechanisms at work in the model, ultimately showing its peculiar but predominant nature.

A few remarks are in order. Of course, our results are based on price-taking firms and our modeling of liquidity-constraints is probably too simple. Adding market power is no problem, although it is not likely that our results would be dramatically altered. Modelling and treating the liquidity constraints more accurately is a much more complicated task, both mathematically and conceptually. We believe that allowing firms to incur into debt to fasten its modernization and compliance to legal standards is a quite decisive issue that should be considered in more comprehensive research. This is our next step.

\section{Appendix}

Proof of Theorem 1: The proof uses standard perturbation techniques of the optimization theory, adjusted for the class of models under study in Hritonenko and Yatsenko (1996, 2005, 2006) and Yatsenko (2004). Let us consider Case (B) first. 
Case (B). If the restriction (13) is inactive, $E^{*}(t)<E_{\max }(t)$ at $t \in \Delta$, then we choose $R, m$, and $v=a^{\prime}$ as the independent unknown variables of the OP. Then, the differential restriction $a^{\prime}(t) \geq 0$ in (14) has the standard form $v(t) \geq 0$. We assume that $R, m$, and $v$ are

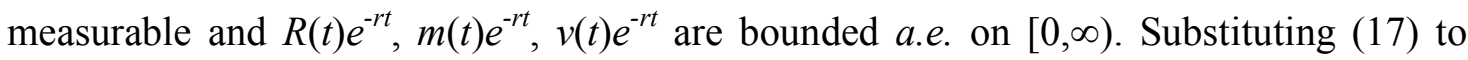
(16), we obtain expression (22) for $\beta(t)$.

We refer to measurable functions $\delta R, \delta m$, and $\delta v$ as the admissible variations, if $R, m, v$, $R+\delta R, m+\delta m$, and $v+\delta v$, satisfy constraints (14)-(15).

Let us give small admissible variations $\delta R(t), \delta m(t)$, and $\delta v(t), t \in(0, \infty)$, to $a, m$, and $R$ and find the corresponding variation $\delta I=I(R+\delta R, m+\delta m, v+\delta v)-I(R, m, v)$ of the objective functional $I$. Using (10)-(13), we obtain that

$$
\begin{gathered}
\delta I=\int_{0}^{\infty} e^{-r t}\left[\int_{a(t)+\delta a(t)}^{t}\left(d b \int_{0}^{\tau}(R(\xi)+\delta R(\xi))^{n} d \xi+B^{d}\right)^{\frac{1}{d}}(m(\tau)+\delta m(\tau)) d \tau\right. \\
\quad-p(t) \int_{a(t)+\delta a(t)}^{t}(m(\tau)+\delta m(\tau)) d \tau-(R(t)+\delta R(t)) \\
\left.\quad-k(t)(m(t)+\delta m(t))\left(d b \int_{0}^{t}(R(\xi)+\delta R(\xi))^{n} d \xi+B^{d}\right)^{\frac{1}{d}}\right] d t \\
-\int_{0}^{\infty} e^{-r t}\left[\int_{a(t)}^{t}\left(d b \int_{0}^{\tau} R^{n}(\xi) d \xi+B^{d}\right)^{\frac{1}{d}} m(\tau) d \tau-p(t) \int_{a(t)}^{t} m(\tau) d \tau-R(t)\right. \\
\left.-k(t) m(t)\left(d b \int_{0}^{t} R^{n}(\xi) d \xi+B^{d}\right)^{\frac{1}{d}}\right] d t
\end{gathered}
$$

where $\delta a(t)=\int_{0}^{t} \delta v(\xi) d \xi$. To prove the Theorem, we shall transform the expression (A1) to the form

$$
\delta I=\int_{0}^{\infty}\left(I_{R}^{\prime}(t) \cdot \delta R(t)+I_{m}^{\prime}(t) \cdot \delta m(t)+I_{v}^{\prime}(t) \cdot \delta v(t)\right) d t+o(\|\delta R\|,\|\delta m\|,\|\delta v\|),
$$

where the norm is $\|f\|=\underset{[0, \infty)}{\operatorname{esssup}}\left|e^{-r t} f(t)\right|$. It will involve several steps. First, using the Taylor expansion $f(x+\delta x)=f(x)+f^{\prime}(x) \delta x+o(\delta x)$ twice, we have that 


$$
\begin{aligned}
& \left(d b \int_{0}^{\tau}(R(\xi)+\delta R(\xi))^{n} d \xi+B^{d}\right)^{\frac{1}{d}} \\
& =\left(d b \int_{0}^{\tau}\left(R^{n}(\xi)+n R^{n-1}(\xi) \delta R(\xi)+o(\delta R(\xi))\right)^{n} d \xi+B^{d}\right)^{\frac{1}{d}} \\
& =\beta(\tau)+b n \beta^{1-d}(\tau) \int_{0}^{\tau} R^{n-1}(\xi) \delta R(\xi) d \xi+\int_{0}^{\tau} o(\delta R(\xi)) d \xi .
\end{aligned}
$$

property

$$
\begin{aligned}
& \text { Next, } \\
& \begin{aligned}
\int_{a(t)+\delta a(t)}^{t} f & \text { using }(\tau) d \tau=\int_{a(t)}^{t} f(\tau) d \tau-\int_{a(t)}^{a(t)+\delta a(t)} f(\tau) d \tau \text { of integrals, we transform (A1) to } \\
\delta I & =\int_{0}^{\infty} e^{-r t}\left[b n \int_{\max \{a(t), 0\}}^{t} m(\tau) \beta^{1-d}(\tau) \int_{0}^{\tau} R^{n-1}(\xi) \delta R(\xi) d \xi d \tau d t\right. \\
& +\int_{0}^{\infty} e^{-r t} \int_{\max \{a(t), 0\}}^{t}(\beta(\tau)-p(t)) \delta m(\tau) d \tau d t \\
& +\int_{0}^{\infty} e^{-r t} \int_{a(t)}^{a(t)+\delta a(t)}(p(t)-\beta(\tau)) m(\tau) d \tau d t-\int_{0}^{\infty} e^{-r t}[\delta R(t)+k(t) \beta(t) \delta m(t)] d t \\
& -\int_{0}^{\infty} e^{-r t} k(t) m(t) \beta^{1-d}(t) \int_{0}^{t} b n R^{n-1}(\xi) \delta R(\xi) d \xi d t+\int_{0}^{\infty} e^{-r t} o(\delta R(t), \delta m(t)) d t,
\end{aligned}
\end{aligned}
$$

where $\max \{a(t), 0\}$ emphasizes that the variations $\delta R(t), \delta m(t)$ are non-zero only on the interval $[0, \infty)$.

Next, we interchange the limits of integration in the second term of (A4) as

$$
\int_{0}^{\infty} e^{-r t}\left[\int_{a(t)}^{t}(\beta(\tau)-p(t)) \delta m(\tau) d \tau d t=\int_{0}^{\infty} \int_{t}^{a^{-1}(t)} e^{-r \tau}(\beta(t)-p(\tau)) d \tau \cdot \delta m(t) d t\right.
$$

in the first term as

$$
\begin{aligned}
& \int_{0}^{\infty} e^{-r t}[\left.\int_{\max \{a(t), 0\}}^{t} m(\tau) \beta^{1-d}(\tau) \int_{0}^{\tau} b n R^{n-1}(\xi) \delta R(\xi) d \xi d \tau\right] d t \\
&=b n \int_{0}^{\infty} \int_{t}^{\infty} \int_{\tau}^{a^{-1}(\tau)} e^{-r \xi} d \xi \cdot m(\tau) \beta(\tau)^{1-d} d \tau \cdot R^{n-1}(t) \delta R(t) d t
\end{aligned}
$$

and in the fifth term similarly. To transform the third term, we use the Taylor expansion $\int_{a(t)}^{a(t)+\delta a(t)} f(t, \tau) d \tau=f(t, a(t))+o(\delta a(t))$. Collecting coefficients of $\delta R, \delta m$, and $\delta a$, we rewrite (A4) as: 


$$
\begin{aligned}
\delta I & =\int_{0}^{\infty}\left[-e^{-r r}+b n \int_{t}^{\infty}\left(\int_{\tau}^{a^{-1}(\tau)} e^{-r \xi} d \xi-e^{-r \tau} k(\tau)\right) \cdot m(\tau) \beta^{1-d}(\tau) d \tau \cdot R^{n-1}(t)\right] \cdot \delta R(t) d t \\
& +\int_{0}^{\infty}\left[\int_{t}^{a^{-1}(t)} e^{-r \tau}(\beta(t)-p(\tau)) d \tau-e^{-r t} k(t) \beta(t)\right] \cdot \delta m(t) d t \\
& +\int_{0}^{\infty} e^{-r t}\left(p(t)-\beta(a(t)) m(a(t)) \cdot \delta a(t) d t+\int_{0}^{\infty} e^{-r t} o(\delta R(t), \delta m(t), \delta a(t)) d t .\right.
\end{aligned}
$$

Finally, recalling $\delta a(t)=\int_{0}^{t} \delta v(\xi) d \xi$, we convert the last expression to

$$
\begin{aligned}
\delta I & =\int_{0}^{\infty}\left[-e^{-r r}+b n \int_{t}^{\infty}\left(\int_{\tau}^{a^{-1}(\tau)} e^{-r \xi} d \xi-e^{-r \tau} k(\tau)\right) \cdot m(\tau) \beta^{1-d}(\tau) d \tau \cdot R^{n-1}(t)\right] \cdot \delta R(t) d t \\
& +\int_{0}^{\infty}\left[\int_{t}^{a^{-1}(t)} e^{-r \tau}(\beta(t)-p(\tau)) d \tau-e^{-r t} k(t) \beta(t)\right] \cdot \delta m(t) d t \\
& +\int_{0}^{\infty} \int_{t}^{\infty} e^{-r \tau}\left(p(\tau)-\beta(a(\tau)) m(a(\tau)) d \tau \cdot \delta v(t) d t+\int_{0}^{\infty} e^{-r t} o(\delta R(t), \delta m(t), \delta v(t)) d t\right.
\end{aligned}
$$

Formula (A5) in notations (21), (24), (25) provides the required expression (A2). The domain (14) of admissible controls $R, m, v$ has the simple standard form $R \geq 0, m \geq 0, v \geq 0$. So, the NCE (23) follows from the obvious necessary condition that the variation $\delta I$ of functional $I$ can not be positive for any admissible variations $\delta R(t), \delta m(t), \delta v(t), t \in[0, \infty)$.

Case (A). If the restriction of (13) is active: $E(t)=E_{\max }(t)$ at $t \in \Delta \subset[0, \infty)$, then we choose $R$ and $m$ as the independent unknowns of the OP. The dependent (state) variable $a$ is uniquely determined from the initial problem

$$
m(a(t)) a^{\prime}(t)=m(t)-E_{\max }(t), \quad a(0)=a_{0},
$$

obtained after differentiating (13). As shown in Hritonenko and Yatsenko (2006), if $E_{\max }{ }^{\prime}(t) \leq 0$, then for any measurable $m(t) \geq 0$, a unique a.e. continuous function $a(t)<t$ exists and a.e. has $a^{\prime}(t) \geq 0$ (see Remark 1 about the possible case $E_{\max }{ }^{\prime}(t)>0$ ). Therefore, the state restrictions $a^{\prime}(t) \geq 0$ and $a(t)<t$ in (14) are satisfied automatically, so we can exclude $a$ from the extremum condition.

Similarly to the previous case, let us give small admissible variations $\delta R(t)$ and $\delta m(t)$, $t \in[0, \infty)$, to $R$ and $m$ and find the corresponding variation $\delta I=I(R+\delta R, m+\delta m)-I(R, m)$ 
of the functional $I$. In this case, the variation $\delta a$ is determined by $\delta m$. To find their connection, let us present (13) as

$$
E_{\max }(t)=\int_{a(t)}^{t} m(\tau) d \tau=\int_{a(t)+\delta a(t)}^{t}(m(\tau)+\delta m(\tau)) d \tau
$$

then

$$
\int_{\max \{a(t), 0\}}^{t} \delta m(\tau) d \tau=\int_{a(t)}^{a(t)+\delta a(t)} m(\tau) d \tau+o(\|\delta m\|,\|\delta a\|)
$$

We will use the above formula (A4) for the variation $\delta I$ as a function of $\delta R, \delta m$, and $\delta a$ and eliminate $\delta a$ from (A4) using (A6). To do that, we rewrite the third term of (D4) as

$$
\begin{aligned}
\int_{0}^{\infty} e^{-r t} & \int_{a(t)}^{a(t)+\delta a(t)}(p(t)-\beta(\tau)) m(\tau) d \tau d t \\
& =\int_{0}^{\infty} e^{-r t}(p(t)-\beta(a(t))) \int_{a(t)}^{a(t)+\delta a(t)} m(\tau) d \tau d t+\int_{0}^{\infty} e^{-r t} \int_{a(t)}^{a(t)+\delta a(t)}(\beta(a(t))-\beta(\tau)) m(\tau) d \tau d t \\
& =\int_{0}^{\infty} e^{-r t}\left(p(t)-\beta(a(t)) \int_{\max \{a(t), 0\}}^{t} \delta m(\tau) d \tau d t+\int_{0}^{\infty} e^{-r t} o(\delta a(t), \delta m(t)) d t\right. \\
& =\int_{0}^{\infty} \int_{t}^{a^{-1}(\tau)} e^{-r \tau}\left(p(\tau)-\beta(a(\tau)) d \tau \cdot \delta m(t) d t+\int_{0}^{\infty} e^{-r t} o(\delta a(t), \delta m(t)) d t\right.
\end{aligned}
$$

by adding $\pm \int_{0}^{\infty} e^{-r t} \beta(a(t)) \int_{a(t)}^{a(t)+\delta a(t)} m(\tau) d \tau d t \quad$ and $\quad$ applying $\quad$ (A6). The integral $\int_{a}^{a+\delta a}(\beta(a)-\beta(\tau)) m(\tau) d \tau$ in (A7) has the order $o(\delta a)$ because $\beta(\tau)$ is continuous.

Substituting (A7) into (A4) and collecting the coefficients of $\delta m$ and $\delta R$, we obtain the expression

$$
\delta I=\int_{0}^{\infty}\left(I_{R}^{\prime}(t) \cdot \delta R(t)+I_{m}^{\prime}(t) \cdot \delta m(t)\right) d t+o(\|\delta R\|,\|\delta m\|)
$$

in the notations (20) and (21). The rest of the proof is identical to Case B.

The Theorem is proved.

Proof of Theorem 2: By Lemma 1, at $n=d$ 


$$
\beta_{\Lambda}(t) \approx \bar{R}(b / C)^{\frac{1}{d}} e^{C t} .
$$

The substitution of (26), (38), and (A9) into equation (29) leads to

$$
b d \bar{M}\left(\overline{\operatorname{R}} e^{C t}\right)^{n-1} \int_{t}^{\infty}\left(\bar{R}\left(\frac{b}{C}\right)^{\frac{1}{d}} e^{C \tau}\right)^{1-n}\left[\frac{e^{-r \tau}-e^{-r(\tau+\bar{E} / \bar{M})}}{r}(1-\theta)-k e^{-r \tau}\right] d \tau=e^{-r t},
$$

and, after integration, to

$$
\frac{d \bar{M} b^{\frac{1}{d}} C^{\frac{n-1}{n}}}{C(1-d)-r}\left[\frac{1-e^{-r \bar{E} / \bar{M}}}{r}(1-\theta)-k\right] e^{-r t}=e^{-r t},
$$

that can be rewritten as (39). Substituting (37), (38), and (A9) to (30) gives

$$
(1-\theta) \int_{t}^{t+\bar{E}}\left[\bar{M}\left[1-e^{C(\tau-\bar{E} / \bar{M})-C t}\right] e^{-r \tau} d \tau \approx k e^{-r t},\right.
$$

which becomes (40) after integration.

Equations (39) and (40) may have a positive solution $C$ and $\bar{M}$ at natural assumptions. In particular, let $r<<1$. Then, presenting the exponents in (40) as the Taylor series, we obtain

$$
\begin{aligned}
& \frac{1}{r}\left[1-1+r \bar{E} / \bar{M}-\frac{1}{2}(r \bar{E} / \bar{M})^{2}+o\left(r^{2}\right)\right] \\
& -\frac{1}{r-C}\left[1-C \bar{E} / \bar{M}+\frac{1}{2}(C \bar{E} / \bar{M})^{2}+o\left(C^{2}\right)-1+r \bar{E} / \bar{M}-\frac{1}{2}(r \bar{E} / \bar{M})^{2}+o\left(r^{2}\right)\right]=\frac{k}{1-\theta}
\end{aligned}
$$

or

$$
\left[\bar{E} / \bar{M}-\frac{r}{2}(\bar{E} / \bar{M})^{2}\right]-\left[\bar{E} / \bar{M}-\frac{1}{2} \frac{1}{r-C}\left(r^{2}-C^{2}\right)(\bar{E} / \bar{M})^{2}\right]+o(r)=\frac{k}{1-\theta}
$$

or

$$
(\bar{E} / \bar{M})^{2}[(r+C)-r]+o(r)=2 k /(1-\theta),
$$

which has the solution $\bar{M}=\bar{E} \sqrt{C / 2 k /(1-\theta)}+o(r)$.

Now, expressing the exponent in (39) as the Taylor series, we obtain

$$
C^{(1-d) / d}[r-C(1-d)]=d b^{1 / d} \bar{M}\left[\bar{E} / \bar{M}-\frac{r}{2}(\bar{E} / \bar{M})^{2}+o(r)-k /(1-\theta)\right] .
$$

Substituting the obtained $\bar{M}$ into (A10) leads to one equation (42) for $C$. To analyze this equation, we use the new variable $x=\sqrt{C}$ and rewrite (42) as

$$
F_{1}(x)=F_{2}(x)
$$


where $\quad F_{1}(x)=x^{2 / d-2}\left(r+x^{2}(d-1)\right), \quad F_{2}(x)=d b^{1 / d} \bar{E}\left[1-\sqrt{\frac{k}{2(1-\theta)}}\left(\frac{r}{x}+x\right)\right]$.

These functions are shown in Figure 3 and are such that $F_{1}(0)=0, F_{1}{ }^{\prime}(x)>0$ at $0<x<\sqrt{r}$, $F_{1}{ }^{\prime}(x)=0$ at $x=\sqrt{r}$, and $F_{2}{ }^{\prime}(x)>0$ at $0<x<\sqrt{r}, F_{2}{ }^{\prime}(x)=0$ at $x=\sqrt{r}$. Also, $F_{2}(x)<0<F_{1}(x)$ at small $0<x<<1$. Therefore, to have a solution $0<x<\sqrt{r}$ to equation (A11), it is necessary and sufficient that $F_{2}(\sqrt{r})>F_{1}(\sqrt{r})$, which leads to the inequality (41). The sufficient condition for the uniqueness of $x$ is $F_{1}{ }^{\prime}(x)<F_{2}{ }^{\prime}(x)$ at $0<x<\sqrt{r}$, which leads to (43).

Finally, let us prove that $c_{\Lambda}(t)>0$ at large $t$.

By $(12), Q_{\Lambda}(t) \approx \bar{R} \bar{M}\left(\frac{b}{C}\right)^{1 / d} \frac{1-e^{-C \bar{E} / \bar{M}}}{C} e^{C t}$. Therefore,

$$
\begin{array}{r}
c_{\Lambda}(t)=(1-\theta) Q_{\Lambda}(t)-k \beta_{\Lambda}(t) m_{\Lambda}(t)-R_{\Lambda}(t)-\bar{E} p(t) \\
\approx \bar{R}\left\{\bar{M}\left(\frac{b}{C}\right)^{1 / d}\left[\frac{1-e^{-C \bar{E} / \bar{M}}}{C}(1-\theta)-k\right]-1\right\} e^{C t}-\bar{E} \bar{P} e^{\gamma t}
\end{array}
$$

Expressing the exponent in (A12) as the Taylor series and using (A10), we obtain

$$
\begin{aligned}
c_{\Lambda}(t) & =\bar{R}\left\{\bar{M}\left(\frac{b}{C}\right)^{1 / d}\left[\frac{\bar{E}}{\bar{M}}(1-\theta)-\frac{C}{2}\left(\frac{\bar{E}}{\bar{M}}\right)^{2}(1-\theta)+o(r)-k\right]-1\right\} e^{C t}-\bar{E} \bar{P} e^{\gamma t} \\
& >\bar{R}\left\{\frac{r-C(1-d)}{C d}(1-\theta)-1\right\} e^{C t}-\bar{E} \bar{P} e^{\vartheta t}=\bar{R} \frac{r-C}{C d}(1-\theta) e^{C t}-\bar{E} \bar{P} e^{\varkappa t}
\end{aligned}
$$

Thus, $c_{\Lambda}(t)>0$ at large enough $t$ for any positive value $\bar{R}$ if $\gamma<C$, and at $\bar{R}>\bar{E} \bar{P} \frac{C d}{(r-C)(1-\theta)}$ if $\gamma=C$. The theorem is proved.

Proof of Lemma 3: We apply the standard method of Lagrange multipliers to take into account the equality-constraint (48), or

$$
R(t)+k(t) \beta(t) m(t)=Q(t)-p(t) E(t), \quad t \in\left[0, t_{\mathrm{k}}\right] .
$$

Let us introduce the Lagrange multiplier $\lambda(t), t \in[0, \infty)$, for the equality (48) and notice that $\lambda(t)=0$ at $t \in\left[t_{\mathrm{k}}, \infty\right)$ because of the complementary slackness condition. Now we minimize the Lagrangian 


$$
L=I+\int_{0}^{\infty}(c(t)-0) \lambda(t) d t
$$

instead of the functional $I$ (10). Providing all transformations as in the proof of Theorem 1 , we arrive to the following expression

$$
\delta L=\int_{0}^{\infty}\left(\hat{I}_{R}^{\prime}(t) \cdot \delta R(t)+\hat{I}_{m}^{\prime}(t) \cdot \delta m(t)+I_{v}^{\prime}(t) \cdot \delta v(t)\right) d t
$$

where

$$
\begin{aligned}
& \widehat{I}_{R}{ }^{\prime}(t)=b n R^{n-1}(t) \int_{t}^{\infty} \beta^{1-d}(\tau) m(\tau)\left[\int_{\tau}^{a^{-1}(\tau)} e^{-r \xi}[1-\lambda(\xi)] d \xi-e^{-r \tau}[1-\lambda(\tau)] k(\tau)\right] d \tau-e^{-r t}[1-\lambda(t)]=0 \\
& \hat{I}_{m}{ }^{\prime}(t)=\int_{t}^{a^{-1}(t)} e^{-r \tau}[1-\lambda(\tau)][\beta(t)-p(\tau)] d \tau-e^{-r t}[1-\lambda(t)] \beta(t) k(t),
\end{aligned}
$$

and $I_{v}{ }^{\prime}(t)$ is given by the same formula (25).

Following the method of Lagrange multipliers, we will choose $\lambda(t)$ from the condition $\hat{I}_{m}^{\prime}(t)=0, t \in\left[0, t_{\mathrm{k}}\right]$. In the new variable $\chi(t)=[1-\lambda(t)] e^{-r t}$, it leads to the formula (55). The expression for $\hat{I}_{R}{ }_{R}(t)$ in the variable $\chi$ is $(54)$ and $\hat{I}_{R}^{\prime}(t)=0$ on $\left[0, t_{\mathrm{k}}\right]$ for interior $R^{*}$. The lemma is proved.

Proof of Theorem 4: We will compare the transition dynamics on $\left[0, t_{\mathrm{k}}\right]$ under two different increasing prices $p(t)$ and $p(t)+\delta p$, where $\delta p=$ const $>0$ on $\left[0, a^{-1}\left(t_{\mathrm{k}}\right)\right]$ or $[0, \infty)$. The perturbation $\delta p$ causes perturbations $\delta a(t), \delta m(t), \delta R(t)$ and $\delta \beta(t)$ of the corresponding optimal $a^{*}(t), m^{*}(t), R^{*}(t)$ and $\beta^{*}(t)$ on interval $\left[0, t_{\mathrm{k}}\right)$ as well as the change $\delta t_{\mathrm{k}}$ of the interval length. The further optimal dynamics on $\left[t_{\mathrm{k}}, \infty\right)$ follows the above Scenario 3. At $t>t_{\mathrm{k}}, \delta R(t)=0$ because $I_{R}(t)$ does not depend on $p$, and $\delta m(t)=0$ because $m^{*}(t)$ is boundary on $\left[t_{\mathrm{k}}, t_{1}\right]$. However,

$$
\delta \beta(t)=b d \beta^{1-d}(t) \int_{0}^{\min \left\{t, t_{k}\right\}} R^{d-1}(\tau) \delta R(\tau) d \tau \text { for all } t \in[0, \infty) .
$$

By (56), $\delta a(t)=\delta p / \beta^{\prime}(a(t))<0$ on $\left[0, t_{\mathrm{k}}\right]$ is determined from the equation $I_{a}^{\prime}(t)=0$ or $\delta a(t)=0$ if $p(t)<\beta_{0}$. The perturbations $\delta m(t), \delta R(t), \delta \beta(t)$, and the auxiliary $\delta \chi(t)$ should not violate equalities (53)-(55). Varying (53), we obtain 


$$
\begin{aligned}
& \delta R(t)+k(t) \beta(t) \delta m(t)-\int_{0}^{t}[\beta(\tau)-p(t)] \delta m(\tau) d \tau+ \\
& +k(t) m(t) \delta \beta(t)-\int_{0}^{t} m(\tau) \delta \beta(\tau) d \tau=-E(t) \delta p
\end{aligned}
$$

Varying (55), we obtain

$$
\begin{aligned}
& \delta \chi(t) k(t) \beta(t)-\int_{t}^{t_{k}} e^{-r(\tau-t)}[\beta(t)-p(\tau)] \delta \chi(t) d \tau= \\
& =\delta \beta(t)\left[\int_{t}^{a^{-1}(t)} \chi(\xi) d \xi-k(t) \chi(t)\right]-\delta p \int_{t}^{a^{-1}(t)} e^{-r \tau} d \tau .
\end{aligned}
$$

Finally, varying (54), we obtain

$$
\begin{aligned}
& -(1-n) \delta R(t) \int_{t}^{t_{k}} \beta^{1-d}(\tau) m(\tau)\left[\int_{\tau}^{a^{-1}(\tau)} \chi(\xi) d \xi-\chi(\tau) k(\tau)\right] d \tau- \\
& -(1-n) \delta R(t) \int_{t_{k}}^{\infty} \beta^{1-d}(\tau) m(\tau)\left[\frac{1-e^{-r\left(a^{-1}(\tau)-\tau\right)}}{r}-k(\tau)\right] e^{-r \tau} d \tau+ \\
& +R(t) \int_{t}^{t_{k}} \beta^{1-d}(\tau)\left[\int_{\tau}^{a^{-1}(\tau)} \chi(\xi) d \xi-\chi(\tau) k(\tau)\right] \delta m(\tau) d \tau+ \\
& +(1-d) R(t) \int_{t}^{\infty} \beta^{-d}(\tau) m(\tau)\left[\int_{\tau}^{a^{-1}(\tau)} \chi(\xi) d \xi-\chi(\tau) k(\tau)\right] \delta \beta(\tau) d \tau= \\
& =\frac{1}{b n} R^{2-n}(t) \delta \chi(t)+R(t) \int_{t}^{t_{k}} \beta^{1-d}(\tau) m(\tau)\left[k(\tau) \delta \chi(\tau)-\int_{\tau}^{t_{k}} \delta \chi(\xi) d \xi\right] d \tau .
\end{aligned}
$$

The proof is clear in the case of small $t_{\mathrm{k}} \beta_{0}<<1$ and $t_{k} \max _{\left[a_{0}, 0\right]} m_{0}<<1$. Then, $\delta \beta(t)=o\left(t_{k}\right)$ by (A14) and by (A16) $\delta \chi(t)=-\delta p \frac{1-e^{-r\left(a^{-1}(t)-t\right)}}{k(t) \beta(t) r}\left[1+o\left(t_{k}\right)\right]<0, t \in\left[0, t_{\mathrm{k}}\right]$, at $\delta p>0$. By (A17),

$$
\delta R(t)=-\delta \chi(t) \frac{R^{2-n}(t)}{(1-n) b n}\left\{\int_{t_{k}}^{\infty} \beta^{1-d}(\tau) m(\tau)\left[\frac{1-e^{-r\left(a^{-1}(\tau)-\tau\right)}}{r}-k(\tau)\right] e^{-r \tau} d \tau\right\}^{-1}\left[1+o\left(t_{k}\right)\right]>0,
$$

on the interval $\left[0, t_{\mathrm{k}}\right]$. Substituting $\delta R$ to (A15), we obtain $\delta m(t)=-\frac{E(t) \delta p+\delta R(t)}{\beta(t) m(t)}\left[1+o\left(t_{k}\right)\right]<0, \quad t \in\left[0, t_{\mathrm{k}}\right] . \quad$ Therefore, $R^{*}(t)$ is larger and $m^{*}(t)$ is smaller at a higher resource price $p(t)$. 
Let us estimate the change $\delta t_{\mathrm{k}}$ of the interval length. The instant $t_{\mathrm{k}}$ is determined from the equality $\int_{a\left(t_{k}\right)}^{t_{k}} m(\tau) d \tau=E_{\max }$. Varying this equality, we obtain $m\left(t_{k}\right) \delta t_{k}=m\left(a\left(t_{k}\right)\right) \delta a\left(t_{k}\right)-\int_{a\left(t_{k}\right)}^{t_{k}} \delta m(\tau) d \tau$. As shown above, $\delta a(t) \geq 0 \quad$ and $\quad \delta m(t)<0$, therefore, $\delta t_{\mathrm{k}}>0$.

In the case of an arbitrary $\left[0, t_{\mathrm{k}}\right]$, we have the system (A14)-(A17) of four non-Volterra integral equations of the second kind for $\delta R(t), \delta m(t), \delta \beta(t), \delta \chi(t), t \in\left[0, t_{\mathrm{k}}\right]$, which is difficult to analyze qualitatively. The only conclusion we can provide is the following. Let $\delta \beta\left(t_{\mathrm{k}}\right) \geq 0$, then by (A15) $\delta R(t)>0$, at least, on some parts of interval $\left[0, t_{\mathrm{k}}\right]$. Now let $\delta \beta\left(t_{\mathrm{k}}\right)<0$, then $\delta \beta(t) \leq 0$ on some final part $\left[t_{\mathrm{c}}, t_{\mathrm{k}}\right], t_{\mathrm{c}}<t_{\mathrm{k}}$, by continuity. Then, by (A16) $\delta \chi(t)<0$ on $\left[t_{\mathrm{c}}, t_{\mathrm{k}}\right]$. By $(\mathrm{A} 17)$

$$
\delta R(t)>-\delta \chi(t) \frac{R^{2-n}(t)}{(1-n) b n}\left\{\int_{t_{k}}^{\infty} \beta^{1-d}(\tau) m(\tau)\left[\frac{1-e^{-r\left(a^{-1}(\tau)-\tau\right)}}{r}-k(\tau)\right] e^{-r \tau} d \tau\right\}^{-1}>0 \quad \text { at } \quad t \rightarrow t_{k},
$$

hence $\delta R(t)>0$, at least, near the end of interval $\left[0, t_{\mathrm{k}}\right]$.

The theorem is proved.

\section{References}

[1] Arrow, K., P. Dasgupta, L. Goulder, G. Daily, P. Ehrlich, G. Heal, S. Levin, K. Mäler, S. Schneider, and B. Walker, Are we consuming too much?, Journal of Economic Perspectives, 18(2004), 147-172.

[2] Benhabib, J. and A. Rustichini, Vintage capital, investment, and growth, Journal of Economic Theory, 55(1991), 323-339.

[3] Boucekkine, R., N. Hritonenko, and Yu. Yatsenko, Optimal firm behavior under environmental constraints, CORE Discussion Paper 2008/24, Université catholique de Louvain, Louvain-la-Neuve, Belgium, 2008; Discussion Paper 2008-11, Department of Economics, University of Glasgow, UK, 2008.

[4] Boucekkine, R. and R. Ruiz-Tamarit, Special functions for the study of economic dynamics: The case of the Lucas-Uzawa model, Journal of Mathematical Economics 44(2008), 33-54.

[5] Boucekkine, R., M. Germain, and O. Licandro, Replacement echoes in the vintage capital growth model, Journal of Economic Theory 74(1997), 333-348. 
[6] Boucekkine, R., F. del Rio, and O. Licandro, Exogenous vs endogenously driven fluctuations in vintage capital growth models, Journal of Economic Theory 88(1999), 161-187.

[7] Feichtinger, G., R. Hartl, P. Kort, and V. Veliov, Environmental policy, the Porter hypothesis and the composition of capital: Effects of learning and technological progress, Journal of Environmental Economics and Management 50(2005), 434446.

[8] Feichtinger, G., R. Hartl, P. Kort, and V. Veliov, Capital accumulation under technological progress and learning: a vintage capital approach, European Journal of Operation Research 172(2006), 293-310.

[9] Hart, R., Growth, environment and innovation- a model with production vintages and environmentally oriented research, Journal of Environmental Economics and Management 48(2004), 1078-1098.

[10] Hartl, R., S. Sethi, and R. Vickson, A survey of the maximum principles for optimal control problems with state constraints, SIAM Review 37(1995), 181-218.

[11] Hicks, J., The theory of wages. McMillan, London, 1932.

[12] Hritonenko, N. and Y. Yatsenko, Modeling and Optimization of the Lifetime of Technologies Kluwer Academic Publishers, Dordrecht, 1996.

[13] Hritonenko, N. and Y. Yatsenko, Turnpike and optimal trajectories in integral dynamic models with endogenous delay, Journal of Optimization Theory and Applications 127(2005), 109-127.

[14] Hritonenko, N. and Y. Yatsenko, Concavity in a vintage capital model with nonlinear utility, Applied Mathematics Letters 19(2006), 267-272.

[15] Jaffe, A., R. Newell, and R. Stavins, Environmental policy and technological change, Environmental and Resource Economics 22 (2002), 41-69.

[16] Kamien, M. and N. Schwartz, Induced Factor Augmenting Technical Progress from a Microeconomic Viewpoint, Econometrica 37 (1969), 668-684.

[17] Krusell, P., Investment-specific R\&D and the decline in the relative price of capital, Journal of Economic Growth 3(1998), 131-141.

[18] Malcomson, J., Replacement and the rental value of capital equipment subject to obsolescence, Journal of Economic Theory 10(1975), 24-41.

[19] Newell, R., A. Jaffee, and R. Stavins, The induced innovation hypothesis and resource-saving technological change, The Quarterly Journal of Economics 114(1999), 941-975.

[20] Parto, S. and B. Herbert-Copley (Eds.), Industrial Innovation and Environmental Regulation, The UN University, Maastricht, 2007.

[21] Porter, M., America's green strategy, Scientific America 264(1991), 4.

[22] Popp, D., Induced innovation and energy prices, American Economic Review 92 (2002), 160-180.

[23] Segerstrom, P., The long-run growth effects of R\&D subsidies, Journal of Economic Growth 5(2000), 277-305 
[24] Solow, R., J. Tobin, C. Von Weizsacker, and M. Yaari, Neoclassical growth with fixed factor proportions, Review of Economic Studies 33(1966), 79-115.

[25] Tsur, Y. and A. Zemel, Scarcity, growth and R\&D, Journal of Environmental Economics and Management 49 (2005), 484-499

[26] Tsur, Y. and A. Zemel, Optimal transition to backstop substitutes for nonrenewable resources, Journal of Economic Dynamics and Control 27 (2003), 551-572.

[27] Yatsenko, Y., Maximum principle for Volterra integral equations with controlled delay time, Optimization 53(2004), 177-187.

[28] Yatsenko, Y., R.Boucekkine, and N.Hritonenko, On explosive dynamics in R\&Dbased models of endogenous growth, to appear in Nonlinear Analysis, 2009, doi 10.1016/j.na.2008.11.068. 


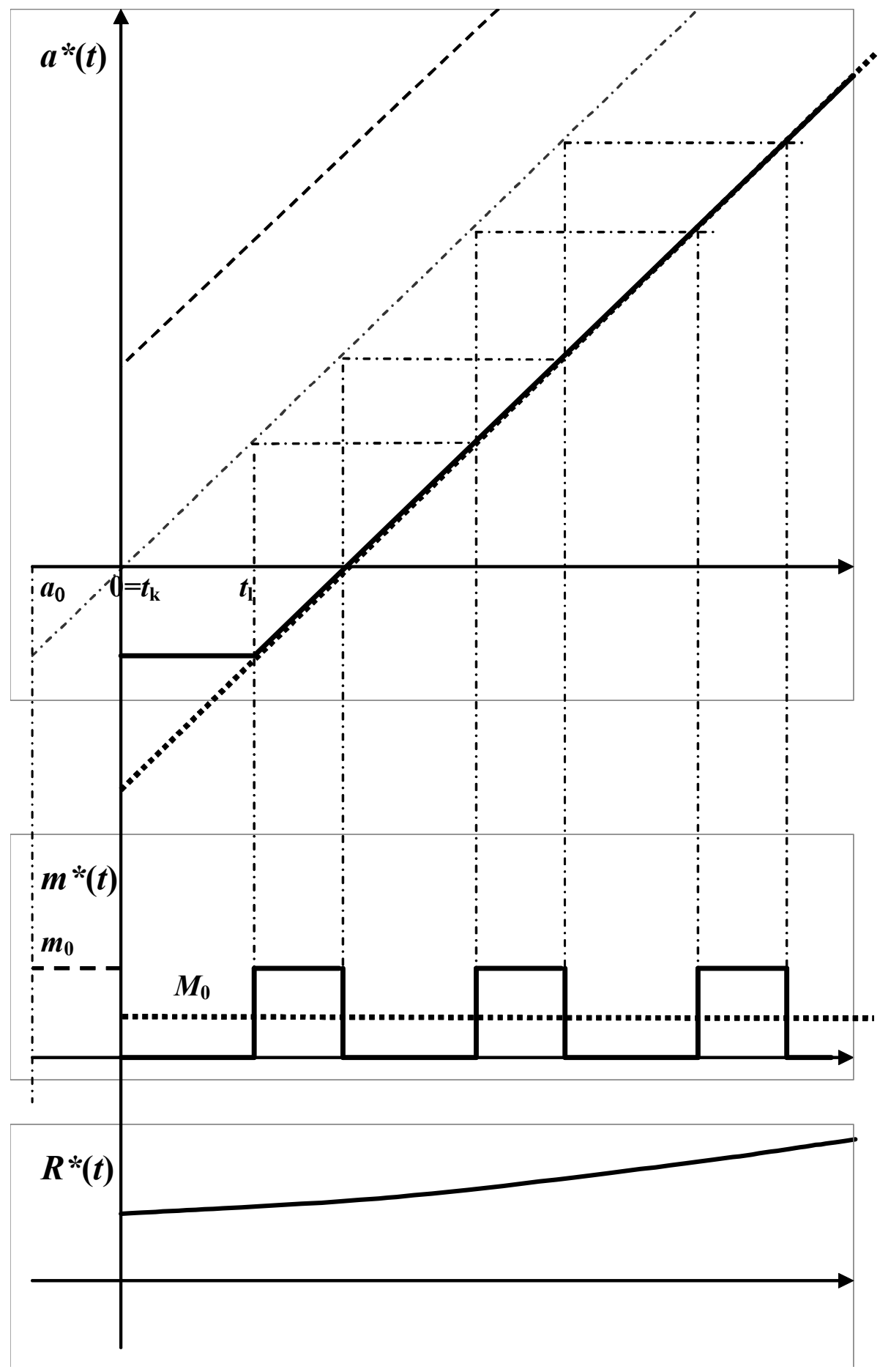

Figure 1. Transition and long-term dynamics under active environment regulation from Example 1 (at specific initial conditions $a_{0}$ and $m_{0}$ ). The dotted lines indicate the BGP regime. The dashed line shows the inverse function $a^{-1}$. 


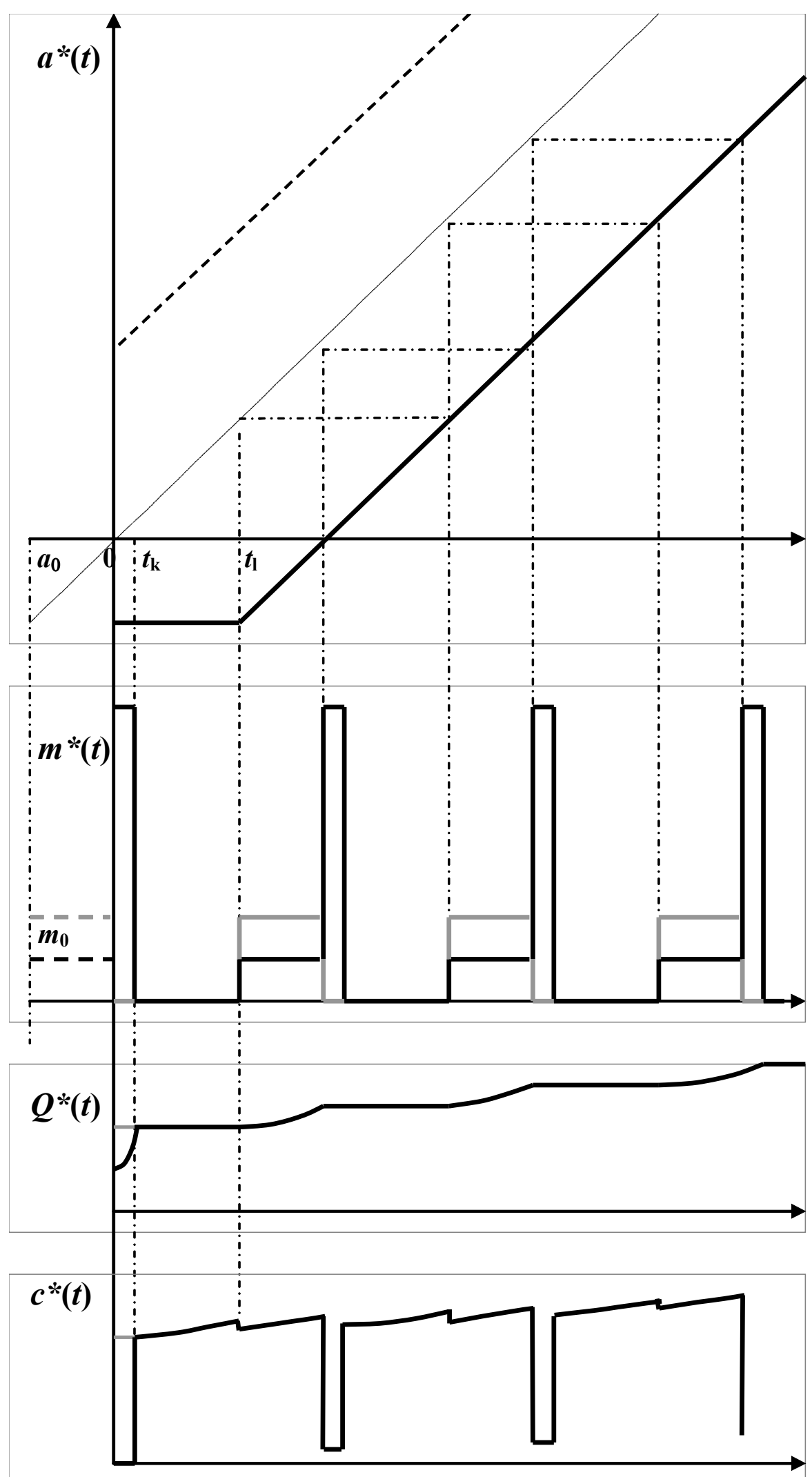

Figure 2. Transition and long-term dynamics under inactive environment regulation from Example 2. The optimal dynamics at active regulation (Example 1) is shown in grey color. 


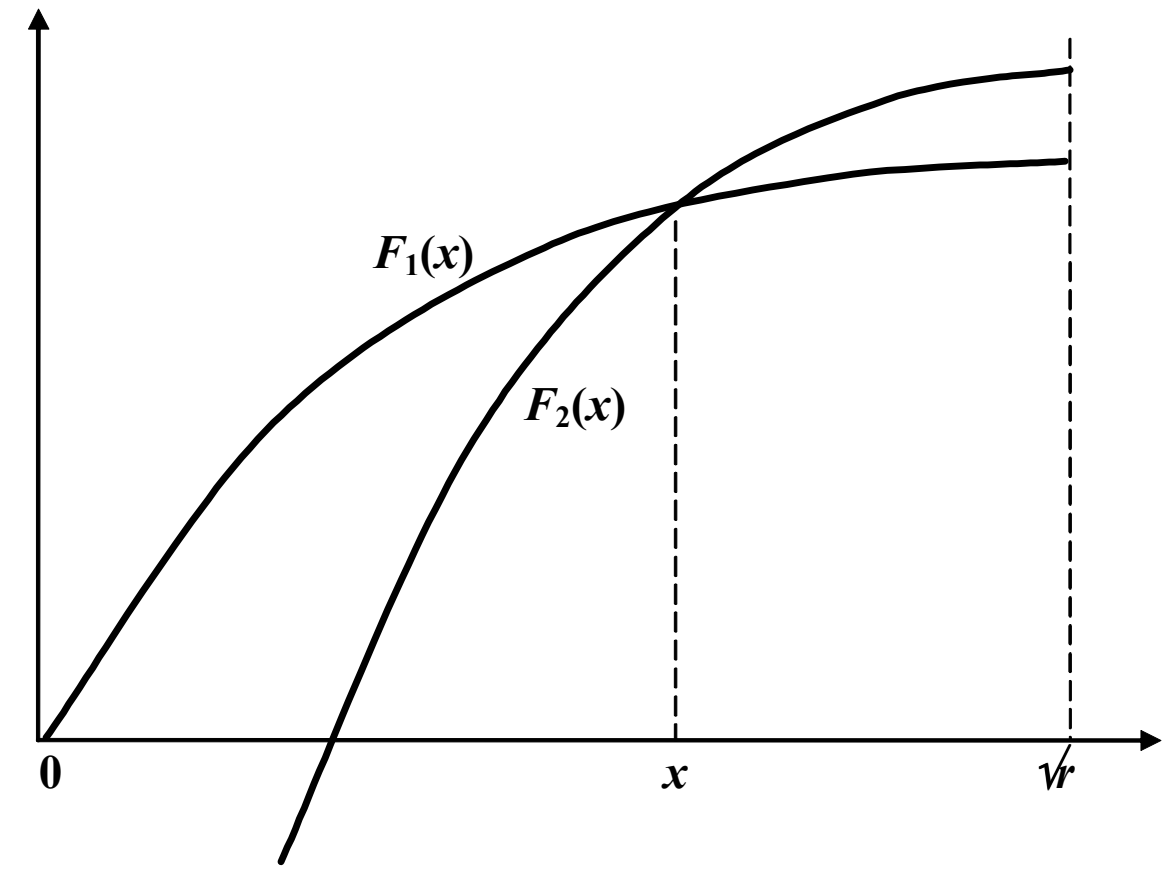

Figure 3. Solving the nonlinear equation (A9) with respect to the unknown $x=\sqrt{C}$. 